\title{
A flux-calibrated, high-resolution atlas of optical sky emission from UVES ${ }^{\star}, \star \star$
}

\author{
R. W. Hanuschik ${ }^{\star \star}$ \\ European Southern Observatory, Karl-Schwarzschild-Str. 2, 85748 Garching, Germany \\ Received 18 April 2003 / Accepted 19 May 2003

\begin{abstract}
This paper presents a flux-calibrated, high-resolution, high-SNR atlas of optical and near-IR sky emission. It provides a complete template of the high-resolution night-sky emission spectrum with the deepest exposures ever obtained from the ground. The data have been acquired by UVES, ESO's echelle spectrograph at the 8.2-m UT2 telescope of the Very Large Telescope (VLT). Raw data stacks with up to 16 hours of integration time have been combined. The spectrum covers the range 3140-10 $430 \AA$ at a resolving power of about 45000 . A total of 2810 sky emission lines have been measured. This high-resolution spectrum is intended to be used for the identification of previously unknown faint sky lines, for simulations of ground based observations where the sky background is important, as a template for checks on the accuracy and stability of the
\end{abstract} \\ wavelength scale, and as a reference for the reduction of spectra of faint objects.
}

Key words. line: identification - atomic data - atmospheric effects - atlases - techniques: spectroscopic

\section{Introduction}

Night-sky (airglow) emission imprints a rich line spectrum, and a faint continuum, on every observed astrophysical spectrum in the optical. The night-sky spectrum is a nuisance for many deep observations. Even at long exposure times, the signal-to-noise ratio (SNR) is insufficient to completely remove the faint emission lines in the blue. On the other hand, many strong lines in the red hide portions of the science data, this effect increasing dramatically towards the infrared. There sky emission lines dominate other emission sources (Rousselot et al. 2000).

It has been realized however that there are also positive aspects to the night-sky emission lines, namely that they provide a template for wavelength self-calibration of the science data (e.g. Osterbrock et al. 1996, 1997).

This paper presents a flux-calibrated night-sky emission atlas at high resolution and high SNR. It extends the existing ISAAC infrared atlas (Rousselot et al. 2000; Lidman et al. 2000) towards the optical range and towards higher spectral resolution.

Input data have been obtained with ESO's echelle spectrograph UVES (Dekker et al. 2000) mounted at the 8.2-m UT2 (Kueyen) telescope of the VLT array on Paranal. The aim of

^ Figures 7-41 are only available in electronic form at http://www.edpsciences.org

Tables 4-9 are only available in electronic form at the CDS via anonymous ftp to cdsarc.u-strasbg.fr $(130.79 .128 .5)$ or via http://cdsweb.u-strasbg.fr/cgi-bin/qcat?J/A+A/407/1157

$\star \star$ Based on observations obtained with the VLT at the European Southern Observatory, Paranal, Chile.

$\star \star \star$ e-mail: rhanusch@eso.org the atlas is to accurately determine night-sky emission line positions and fluxes. These data are potentially useful for deep spectroscopy of point and extended sources, but also for other applications, e.g. aeronomy. The advantages of the present atlas over the existing data from the Keck $10 \mathrm{~m}$ telescope (Osterbrock et al. 1996, 1997) are the flux calibration, the higher number of line positions, the higher SNR and the extension to the blue. It also provides a useful supplement of the recent $U B V R I$ Paranal night sky survey of Patat (2003).

\section{Acquisition}

The sky emission spectrum has been constructed from archival and public deep science exposures. The exposures have been selected using the following criteria:

- exposure time at least 1 hour;

$-2 \times 2$ binning;

- seeing $1^{\prime \prime}$ or better;

- minimal moon contamination (fractional lunar illumination $<0.05$, and moon distance $>90^{\circ}$ );

- object well centred and point-like.

Exposure time, binning and moon constraints were dictated by the quest for highest-possible sensitivity. The seeing constraint is important because the slit length was quite limited. In the blue, the standard slit length is $10^{\prime \prime}$ or about 18 binned pixels. In the red, the slit is $12^{\prime \prime}$ long or 30 binned pixels. A longer slit would have been more favourable for this project but is normally not chosen for science observations, to provide sufficient inter-order space. Therefore the UVES sky spectra had 
Table 1. Sample of input data.

\begin{tabular}{|c|c|c|c|c|c|c|}
\hline Setting* & Range $(\AA)$ & $\begin{array}{l}\text { Number of spectra } \\
\text { (month/year) }\end{array}$ & Airmass & Seeing & $\begin{array}{l}\text { Fractional lunar } \\
\text { illumination }\end{array}$ & $\begin{array}{l}\text { Total exposure } \\
\text { time }(\mathrm{h})\end{array}$ \\
\hline 346 DI1 $2 \times 2$ & $3140-3760$ & $\begin{array}{l}7(06 / 2001)+ \\
9(08 / 2001)\end{array}$ & $\begin{array}{l}1.024-1.336 \\
\text { (mean: } 1.143 \text { ) }\end{array}$ & $\begin{array}{l}0.45-1.09 \\
\text { (mean: } 0.68 \text { ) }\end{array}$ & $\begin{array}{l}0.002-0.048 \\
\text { (mean: } 0.018 \text { ) }\end{array}$ & 16.0 \\
\hline 437 DI $22 \times 2$ & $3740-4860$ & $\begin{array}{l}9(06 / 2001)+ \\
3(07 / 2001)\end{array}$ & $\begin{array}{l}\text { 1.021-2.047 } \\
\text { (mean: } 1.241 \text { ) }\end{array}$ & $\begin{array}{l}0.39-0.76 \\
\text { (mean: } 0.55 \text { ) }\end{array}$ & $\begin{array}{l}0.000-0.050 \\
\text { (mean: } 0.023 \text { ) }\end{array}$ & 13.2 \\
\hline 580 DI1 $2 \times 2$ & $\begin{array}{l}\text { L: 4810-5770, } \\
\text { U: 5830-6790 }\end{array}$ & $7(06 / 2001)$ & $\begin{array}{l}\text { 1.024-1.321 } \\
\text { (mean: } 1.144 \text { ) }\end{array}$ & $\begin{array}{l}0.62-0.83 \\
\text { (mean: } 0.72 \text { ) }\end{array}$ & $\begin{array}{l}0.002-0.035 \\
\text { (mean: } 0.014 \text { ) }\end{array}$ & 7.0 \\
\hline 860 DI $22 \times 2$ & $\begin{array}{l}\text { L: } 6700-8560, \\
\text { U: } 8600-10430\end{array}$ & $9(06 / 2001)$ & $\begin{array}{l}\text { 1.021-1.412 } \\
\text { (mean: } 1.108 \text { ) }\end{array}$ & $\begin{array}{l}0.40 . .0 .57 \\
\text { (mean: } 0.50 \text { ) }\end{array}$ & $\begin{array}{l}0.000-0.050 \\
\text { (mean: } 0.025 \text { ) }\end{array}$ & 9.0 \\
\hline
\end{tabular}

* Central wavelength in $\mathrm{nm}$, dichroic filter, binning; $\mathrm{L}=$ lower red $\mathrm{CCD}, \mathrm{U}=$ upper red CCD.

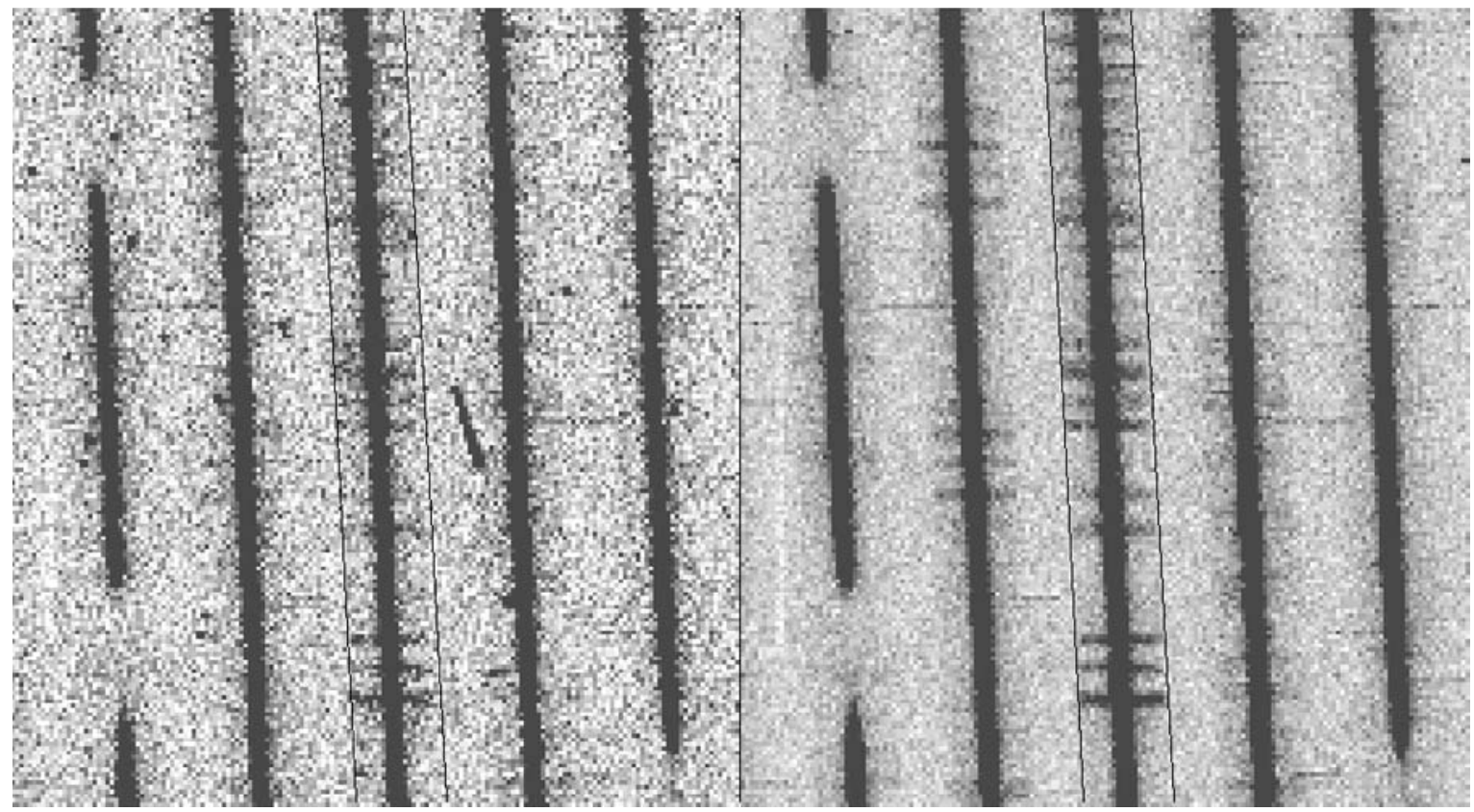

Fig. 1. Left: Single raw file (1 hour exposure). Right: Stack from seven input raw files (total exposure time 7 hours). The setting $346 \_D I 1 \_2 \times 2$ has been used here. Echelle orders run almost vertically, with wavelength increasing downwards and to the right. The two black lines mark the sky-exposed part of one specific order. The dark continuum is stellar flux. The sky emission lines, as monochromatic images of the entrance slit, mark the slit extension. The inter-order space (bright) can clearly be distinguished from the slit-illuminated CCD areas on the stacked frame.

to be extracted from quite a limited range along the spatial coordinate of the slit, unlike to long-slit spectra (see Fig. 1). Any degrading by bad seeing would have polluted the stellar source into the sky region, especially in the blue.

For the same reason it has been very important to find wellcentred spectra from point-like sources. Input frames were visually checked for proper centring and against contamination by multiple sources or extended background emission.

The slit width in all spectra was $1.0^{\prime \prime}$. This is a reasonable trade-off between highest SNR and highest spectral resolution. The slit width of $1.0^{\prime \prime}$ translates into resolving power $R=45000$ in the blue, and 43000 in the red. Selected input data are summarized in Table 1 .

All spectra have been obtained in Service Mode runs. Most data were collected around the new moon of June 2001 (nights 20-22/06/2001). Some spectra have been added from the new moon periods of July 2001 (nights 18-21/07/2001) and August 2001 (nights 16-19/08/2001).

UVES has a mosaic of one blue and two red CCDs. Two blue standard settings were selected (central wavelength $346 \mathrm{~nm}$, dichroic filter \#1 and $437 \mathrm{~nm}$, dichroic \#2), and two red standard settings $(580 \mathrm{~nm}$, dichroic \#1, and $860 \mathrm{~nm}$, dichroic \#2), all in binned mode $(2 \times 2)$. The data cover the whole wavelength range accessible to UVES.

\section{Reduction}

The spectra have been reduced with the UVES pipeline (version 1.7.0) (Ballester et al. 2000), using pipeline-processed master calibration data from the same period. Two steps were necessary:

1. All spectra per setting and per period were stacked and combined, in order to remove cosmic ray hits and 

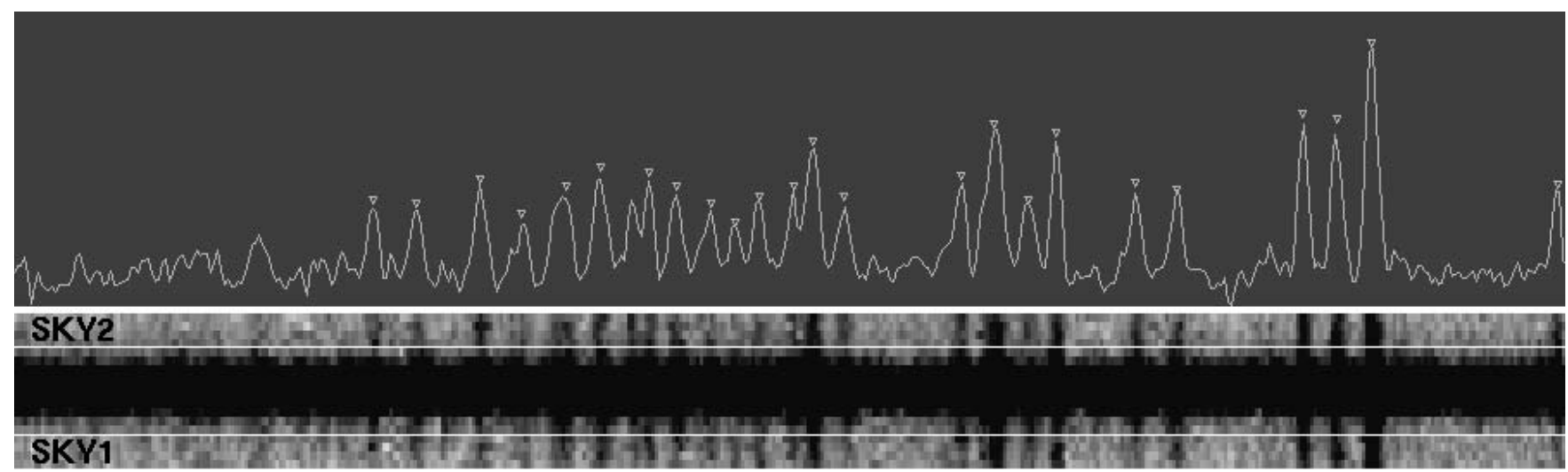

Fig. 2. 2D extracted (bottom) and 1D extracted spectrum (top). The central order marked in Fig. 1 has been selected here. Wavelength scale increases towards the right. The two sky windows are marked. The collapsed 1D sky spectrum has been constructed from these two windows. The features accepted and measured as sky emission lines are marked by open triangles.

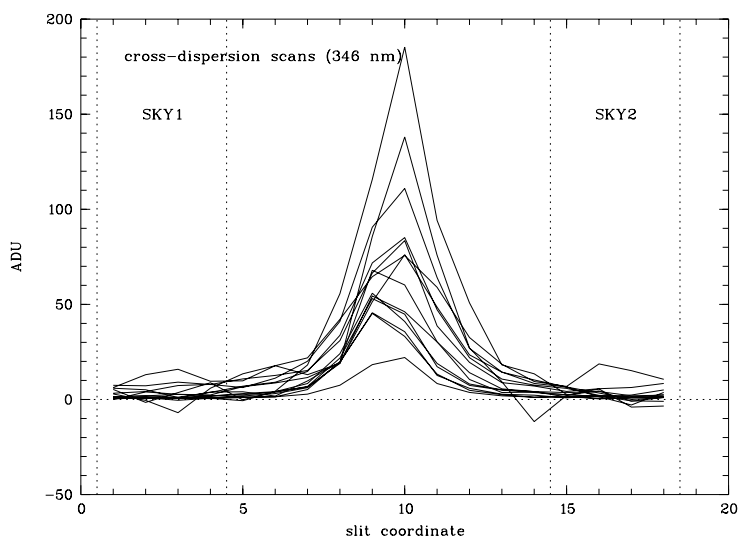

Fig. 3. Cross-dispersion scans (setting 346_DI1_2 2 2). One scan every $50 \AA$ in slit direction is shown. The two areas defined by the broken lines define the two extraction windows for the sky spectrum (SKY1, SKY2). Their width is 4 binned pixels (2") each, to be safe for the seeing wings of the object signal.

improve the SNR (see Fig. 1). This step made use of the uves_cal_mkmaster recipe which is usually employed to stack sets of raw BIAS or FLAT data. Cosmic ray removal was very efficient.

2. The stacked science data were then reduced with the standard recipe for science reduction, uves_obs_scired. Extraction mode was "2D", which means the data were debiased, flattened, wavelength-calibrated, merged, and resampled to a two-dimensional wavelength- $Y$ grid where $Y$ is the vertical slit coordinate (Fig. 2).

The blue data come from two different epochs (Table 1), with two different grating orientations. They were co-added per period and reduced separately. Only after full reduction were the results averaged into the final result. All data from within the same period (covering a few days) were reduced with the same wavelength calibration and flat field files.

In the blue, two sky windows of 4 binned pixels each $\left(2^{\prime \prime}\right)$ were finally extracted, located at the upper and lower boundaries (SKY1 and SKY2, see Fig. 2), thereby avoiding contamination from the signal source. The width of the sky windows was chosen empirically. Figure 3 shows cross-dispersion profiles for the bluest data. The final 1D sky spectrum was obtained from collapsing and averaging the two sky windows.

In the red, a regular pipeline-provided 1D extraction of the science spectrum delivered extracted and averaged sky windows as a by-product. This product was chosen as $1 \mathrm{D}$ spectrum since it has a better merging algorithm for the echelle orders.

Both the extracted (1D) and rectified but otherwise unextracted (2D) results was used for the following line measurements because both have their specific pros and cons. The 2D spectra are superior for line measurements and reliability assessment. The pipeline-delivered results have however their limitations, e.g. flat-field artefacts, a crude order merging algorithm and a narrower wavelength coverage. The 1D spectra (obtained from averaging the 2D data in the blue, and from direct pipeline extraction of the input data in the red) provide better emission line plots and higher SNR but limited capacity for recognizing noise and artefacts.

\section{Flux calibration}

\subsection{Master response curves}

The extracted spectra still carry some large-scale signature of the spectrograph, namely the ratio of sky emission and flat field spectral slopes. To obtain calibrated fluxes, master response curves were used. These were derived from a large set of flux standard star measurements obtained over roughly the same period as the input spectra ${ }^{1}$.

A typical UVES night sees standard star measurements with a $10^{\prime \prime}$ slit in the evening and in the morning twilight. The UVES pipeline creates from each input standard star frame a response curve by dividing the extracted signal into the tabulated physical flux of the standard star. These response curves need to be corrected for exposure time, binning, gain and extinction. Furthermore they show stellar spectral features which need to be treated properly before they are compared to the tabulated flux values. These values typically come on a $50 \AA$ grid.

\footnotetext{
${ }^{1}$ Find more information about the UVES master response curves, including downloads, at http://www . eso.org/qc/UVES/qc/std_qc1.html
} 


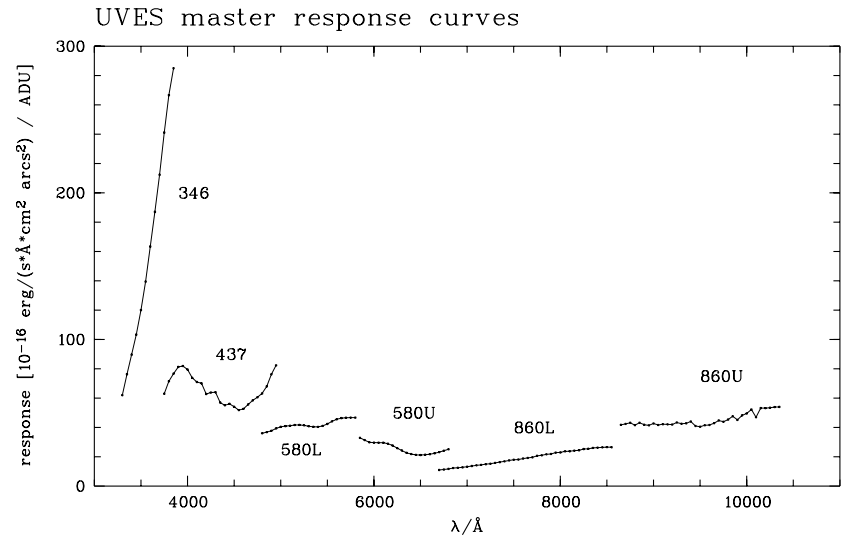

Fig. 4. Master response curves selected for the flux calibration of the sky emission spectrum.

It is also obvious that the quality of the nights, and of the standard star fluxes, vary. It is therefore necessary to carefully select night-by-night response curves. The results of the correction and selection steps are called the master response curves. These provide a high-quality flux calibration of the relative slope of the spectrum. The absolute scale of the flux-calibrated spectrum may still suffer from systematic effects like slit losses and variability of the photometric quality in a night.

For the flux calibration of the UVES sky spectrum, a set of master response curves was selected which is valid for the period 01/06/2001 until 30/09/2001 (Fig. 4).

\subsection{Flux calibration of sky emission spectra}

The following expression has been used for the flux calibration of the sky emission spectra:

$F_{\text {sky }}(\lambda)=f_{\text {sky }}(\lambda) \cdot E(\lambda) \cdot \epsilon_{\text {conv }} / T_{\text {exp }} \cdot M_{\mathrm{RSP}}(\lambda) / s_{\mathrm{px}}^{2}$

where $F$ and $f$ denote the physical (calibrated) and the extracted (uncalibrated) flux of the sky spectrum, respectively; $E(\lambda)$ is the extinction correction; $\epsilon_{\text {conv }}$ is the CCD conversion factor (=1/gain); $T_{\exp }$ is the average exposure time per input spectrum; $s_{\mathrm{px}}$ is the pixel scale, and $M_{\mathrm{RSP}}(\lambda)$ is the master response curve. The pixel scale enters here since for extended sources like sky emission, $F_{\text {sky }}(\lambda)$ is the surface flux. Table 2 has the parameters mean exposure time, conversion factor and pixel scale for each set-up used here.

The flux calibration of the sky emission spectra has some specific problems:

Extinction correction. The major part of the sky emission spectrum is due to $\mathrm{OH}$ Meinel bands and arises in atmospheric heights of typically $90 \mathrm{~km}$ (Leinert et al. 1998). Light emitted at those altitudes can be expected to suffer from essentially the same extinction as light from extraterrestrial sources. However, the emission layer of finite distance and extent introduces a special geometric effect. The optical path length through a layer of thickness $l_{0}$ along a line of sight at airmass $\mathrm{X}$ increases with $\mathrm{X}$, $l \approx l_{0} \cdot X$ (Roach \& Meinel 1955; Garstang 1989). This causes the night sky emission to brighten towards the horizon.

This is applicable, however, for only a fraction $f$ of the continuum sky emission which is of atmospheric origin, while the
Table 2. Numbers for flux calibration.

\begin{tabular}{l|lll}
\hline \hline Setting* & $\begin{array}{l}T_{\exp } \\
\left(\mathrm{s}^{-1}\right)\end{array}$ & $\epsilon_{\mathrm{conv}}$ & $\begin{array}{l}S_{\mathrm{px}} \\
\left({ }^{\prime \prime} / \mathrm{px}\right)\end{array}$ \\
\hline 346 DI1 2 2 & 3600 & 0.60 & $0.246 * 2$ \\
437 DI2 2×2 & 3876 & 0.60 & $0.246 * 2$ \\
580 DI1 2 2 & 3600 & 0.52 & $0.182 * 2$ \\
860 DI2 2 2 & 3600 & 0.52 & $0.182 * 2$ \\
\hline
\end{tabular}

* Central wavelength in $\mathrm{nm}$, dichroic filter, binning.

remaining fraction $(1-f)$ is from diffuse extraterrestrial sources and does not show that effect (Patat 2003).

Assumptions on $f$ are very uncertain. It is also well known that sky emission is highly variable (see Patat 2003). Therefore only a standard extinction correction was applied which focuses on the leading effect, namely the extinction which is strongly chromatic, especially in the blue:

$E(\lambda, X)=10^{-0.4 e(\lambda) X}$

The standard La Silla extinction law e, extrapolated beyond $9000 \AA$ A , has been used (Tüg 1980) which takes into account Rayleigh scattering, ozone and aerosol absorption. The mean airmass values from Table 1 have been used.

Neglecting the geometric brightening in principle leads to an overcorrection of extinction, and hence the sky emission flux. Using the $U B V R I$ extinction coefficients for Paranal from Patat's Table A.1, the mean airmass values from Table 1, and $f=0.6$ (Patat's Appendix C), the effect of the overshoot can be estimated. The result is that on average the corrected fluxes may be too high by about 0.10 mag (less in the red, more in the 437 setting with its higher average airmass), with no strong wavelength dependence. We will ignore that potential effect in the following.

Contamination. As much care as possible has been taken to select data from really dark time (see Sect. 2). Also contamination has been avoided as far as possible, either from the seeing wings of the scientific objects or from extraterrestrial diffuse emission (such as extended HII regions). All input raw frames have been inspected to have seeing better than $1^{\prime \prime}$. The median stacking process is expected to remove background emission outliers. Residual contamination, if present at all, is likely to be very small.

Intrinsic variability. Sky emission lines are well known to be variable on various time and spatial scales. The input data used here have been collected from different nights and from long exposures. The measured line fluxes are therefore an average only.

Setting 346_DI1 $2 \times 2$. The master response curve for this setting is established redwards of $3300 \AA$ only, because of the standard stars used during the validity period. The curve used here has been extrapolated bluewards towards $3100 \AA$ using a second-order polynomial. 


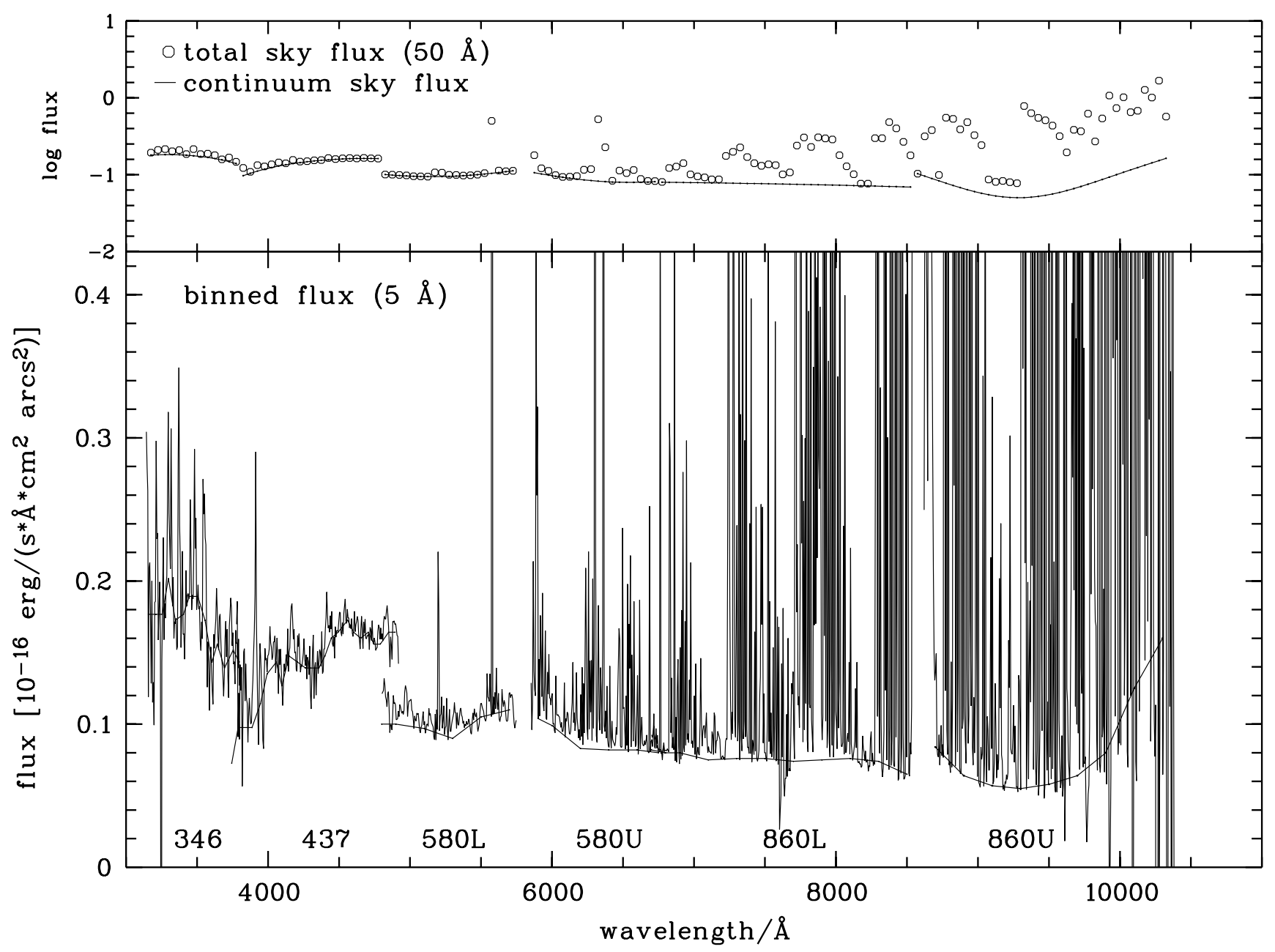

Fig. 5. Strongly averaged UVES sky emission spectrum. Bottom: flux sampled into $5 \AA$ bins. The continuum flux, indicated by the solid line, is roughly constant at about $1-2 \times 10^{-17} \mathrm{cgs}$ units ( $\mathrm{erg} \mathrm{s}^{-1} \AA^{-1} \mathrm{~cm}^{-2} \operatorname{arcs}^{-2}$ ). The settings used are labelled. Top: the total sky emission flux (line plus continuum) averaged across $50 \AA$ bins, and the continuum flux plotted as solid line. The flux scale is logarithmic, with the same flux units as in the bottom panel.

Setting 437_DI2 $2 \times 2$. The flux calibration in this setting might be less certain than in the other settings because of the intrinsic uncertainties of the master response curve in the Balmer jump region.

\subsection{Product spectra}

The flux-calibrated UVES sky emission spectrum has been collected in two forms:

- the flux calibrated and extracted spectrum (1D);

- the flux calibrated two-dimensional spectrum (2D).

In either case the result spectra come in six separate wavelength regions: one for each of the two blue settings (central wavelengths 346 and $437 \mathrm{~nm}$ ), and four for the two red settings, each of which splits into two spectra because of the two red CCDs. The six sub-spectra have not been merged into one single spectrum since that would compromise too much the bin size of the data.

The bottom part of Fig. 5 shows the flux-calibrated nightsky spectrum, sampled to $5 \AA$ to illustrate the continuum slope.
Steps between certain portions of the spectrum presumably come from uncertainties in the extraction of the faint sky continuum flux. The full spectrum is plotted in Figs. 7-41.

\section{Line measurements}

\subsection{Selecting emission lines}

Before discussing the sky emission spectrum in more depth, we want to focus on measurements of the emission lines, in order to disentangle continuum and line emission.

Apart from providing the flux-calibrated sky emission spectrum, the second purpose of this paper is to derive accurate emission line positions and strengths. For that purpose the 2D spectra, the extracted SKY1 and SKY2 spectra, and their error (expressed as rms of the difference spectra) have been used. We aim to provide input data for spectroscopic identifications. A starting point for these may be found e.g. in Osterbrock et al. (1996, 1997), in Abrams et al. (1994), and in Rousselot et al. (2000).

It turned out that the main challenge is not to find emission lines but to reject false lines due to e.g. reduction or CCD 
artefacts, residual cosmic rays or just noise. The following criteria had to be met for a line to be accepted for the catalogue:

- Ideally the emission line should be visible as a vertical bar in the $2 \mathrm{D}$ spectrum, with the minimum width set by the resolving power. This works fine for stronger lines and is also powerful for identifying and rejecting artefacts of the reduction process, or residual cosmic rays.

- For fainter lines, the 1D spectra and their error function have been used. The latter should not increase around a line candidate. The extracted SKY1 and SKY2 spectra should show the same structure with about the same strength. This criterion protects against misidentifications of residual cosmic rays and also in noisy spectral regions. Problems due to noise are common at the beginning of each echelle order. This criterion is very powerful, in particular when the first criterion starts failing for faint features.

These two criteria in combination are very efficient for the detection of sky emission lines which essentially should be monochromatic images of the slit, e.g., those many peaks in the blue spectrum that do not have an identification bar have clearly failed at least one criterion.

The list of measured lines can be claimed as being almost complete at the resolution and SNR level of the input data. Those spectral regions corresponding to the beginning of a new echelle order may still hide some features in enhanced noise. There are also small spectral gaps of a few $\AA$ between the redmost orders beyond $9760 \AA$ (see Figs. 36-38), and two larger gaps of about 40-60 $\AA$ at the central wavelengths of the two red settings (5800 and $8600 \AA$ ) due to the physical gaps between the two red CCDs in the mosaic.

Once a feature had been assessed as a valid emission line, a Gaussian fitting routine was employed to obtain the central wavelength, the central flux, and the FWHM of the fit. These three parameters (called CENTER, FLUX, and FWHM) are listed in the result tables, together with the line intensity from uncalibrated spectra (INT).

The red upper CCD suffers from a leaking column causing broad quasi-emission in the echelle order. These features have been easily recognized due to their known position and atypical width. They are marked by squared crosses in the plots.

Line blends that would be considered as resolved with a simple cursor-marked approach are still a blend for the Gaussian fit procedure unless two peaks are clearly separated. Hence some of the lines catalogued here are indeed marginally resolved blends with a FWHM in excess of the resolution limit.

\subsection{Accuracy of line positions}

The accuracy of the line positions measured is determined by the internal accuracy of the wavelength scale, and by the accuracy of the Gaussian fitting.

The internal accuracy of the wavelength scale is measured by the rms of the difference between the global dispersion solution and the arclamp emission lines. The mean rms values for binned data range between $4.7 \mathrm{~m} \AA$ for the $346 \mathrm{~nm}$ setting and

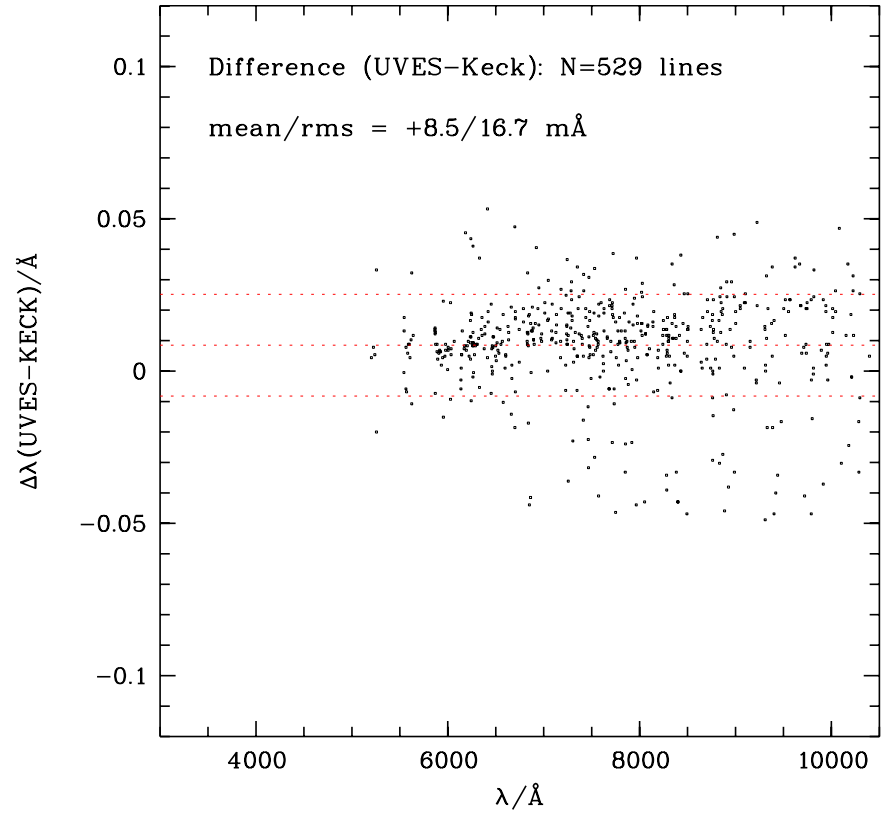

Fig. 6. Residuals of wavelength determinations between the Keck atlas and the UVES atlas. 529 lines redwards of $5200 \AA$ have been measured, with a mean difference of $8 \mathrm{~m} \AA$ and a standard deviation of $17 \mathrm{m \AA}$.

$10 \mathrm{~m} \AA$ for the $860 \mathrm{~nm}$ setting ${ }^{2}$. These numbers translate into $1 / 15-1 / 20$ resolution elements.

The FWHM values of the measured lines cluster very closely around the nominal values expected from the resolving power. This means that the process of averaging the data from several days, and from different epochs in the case of the blue data, has not introduced systematic shifts degrading the spectral resolution.

A simple cross-check of the precision of the line positions is the comparison between the line positions as listed in the Keck atlas and in the present atlas. The difference

$d=\lambda_{\mathrm{UVES}}-\lambda_{\mathrm{Keck}}$

has been measured for $N=529$ lines redwards of $5200 \AA$. The result is plotted in Fig. 6. To find the most likely associations between Keck and UVES line positions in an automatic procedure, a numerical window has been used, and all lines outside the window have been rejected. The window size has been $0.05-0.10 \AA$. The result is

$<d>=+8 \pm 17 \mathrm{m \AA}$.

The error given is the likely error of a single line position. To assess the accuracy of the wavelength calibration achievable with the line lists presented here, let us focus on the region 7000-7100 $\AA$ having a rich sky emission spectrum with 58 measured emission lines in the present atlas. The rms error of the wavelength calibration in that region would be about $2 \mathrm{m \AA}$.

\footnotetext{
${ }^{2}$ http://www. eso.org/qc/UVES/qc/resolution_qc1.html, select "dispersion rms".
} 
Table 3. Measured sky emission lines.

\begin{tabular}{|c|c|c|c|c|}
\hline Setting* & Range $(\AA)$ & $\begin{array}{l}\text { Typical level } \\
\text { of sky continuum }{ }^{1}\end{array}$ & $\begin{array}{l}\text { Number of } \\
\text { sky emission lines }\end{array}$ & $\begin{array}{l}\text { Average contribution } \\
\text { from sky emission lines }\end{array}$ \\
\hline 346 DI1 $2 \times 2$ & $3140-3760$ & 0.17 & 373 & 0.02 \\
\hline 437 DI $22 \times 2$ & $3740-4860$ & 0.14 & 565 & 0.01 \\
\hline 580 DI1 $2 \times 2$ & L: $4800-5770$ & 0.09 & 88 & 0.02 \\
\hline 580 DI1 $2 \times 2$ & U: 5830-6790 & 0.10 & 172 & 0.04 \\
\hline 860 DI $22 \times 2$ & L: $6700-8560$ & 0.08 & 1014 & 0.01 \\
\hline 860 DI $22 \times 2$ & U: 8600-10 430 & 0.07 & 589 & 0.40 \\
\hline
\end{tabular}

* Central wavelength in nm, dichroic filter, binning; L: lower red CCD, U: upper red CCD.

${ }^{1}$ In $10^{-16}$ cgs units.

2 Averaged across $50 \AA$, same units as above.

\section{Results: Flux-calibrated sky emission spectrum}

\subsection{Emission line spectrum and tables}

Figures 7-41, published electronically, show the extracted UVES sky emission spectrum. Tables 4-9, available at the CDS, list the positions, widths and fluxes for all sky emission lines.

The spectrum is plotted in 20 or $30 \AA$ panels, with starting and end wavelength denoted at the borders. The figures are arranged as follows:

- Figs. 7-10: $\lambda \lambda 3140-3760 \AA$ (setting 346 DI1 $2 \times 2$ );

- Figs. 11-17: $\lambda \lambda 3740-4860 \AA$ (setting 437 DI2 $2 \times 2$ );

- Figs. 18-21: $\lambda \lambda 4810-5770 \AA$ (setting 580 DI1 $2 \times 2$, lower red CCD);

- Figs. 22-25: $\lambda \lambda 5830-6790 \AA$ (setting 580 DI1 $2 \times 2$, upper red CCD);

- Figs. 26-33: $\lambda \lambda 6700-8560 \AA$ (setting 860 DI2 $2 \times 2$, lower red $\mathrm{CCD})$;

- Figs. 34-41: $\lambda \lambda 8600-10040 \AA$ (setting 860 DI $22 \times 2$, upper red $\mathrm{CCD}$ ).

The plots redward of $5200 \AA$ have full circles marking emission lines with their identification in the Keck atlas (Osterbrock et al. 1996).

The plots redward of $6000 \AA$ have markings for the positions of identified $\mathrm{OH}$ lines in the Rousselot et al. (2000) list. Line positions below $9975 \AA$ have been taken from the extended list (Lidman et al. 2000). All positions have been transformed from vacuum to air using the Edlén (1966) formula:

$$
\begin{aligned}
\lambda_{\text {air }} & =\lambda_{\text {vacuum }} / n, \\
n & =1+10^{-8}\left(8342.13+\frac{2,406,030}{130-\sigma^{2}}+\frac{15,997}{38.9-\sigma^{2}}\right),
\end{aligned}
$$

where $\sigma=1 / \lambda$ and $\lambda$ is measured in $\mu$.

\subsection{Line and continuum fluxes}

The bottom panel of Fig. 5 shows the complete extracted sky emission spectrum as registered in the six set-ups, sampled into $5 \AA$ bins. The averaged continuum flux is marked by the solid lines. Steps between certain portions illustrate the possible uncertainties of the flux calibration. The continuous (extinction-free) sky emission is roughly constant between about $2 \times 10^{-17}$ cgs units (erg s$\AA^{-1} \AA^{-1} \mathrm{~cm}^{-2} \operatorname{arcs}^{-2}$ ) in the UV, and $1 \times 10^{-17} \mathrm{cgs}$ in the red (Table 3 ).

The top part of Fig. 5 shows the total sky emission flux averaged over $50 \AA$, and the continuum flux separately. Below $5500 \AA$, sky emission lines generally do not contribute significantly to the total sky emission flux. Although locally emission lines are frequent, they are all faint. Between $5500 \AA$ and $6500 \AA$, the strong atomic sky emission lines of [O I] ( $\lambda \lambda 5577.35,6300.30$, and 6363.78 $\mathrm{\AA}$ ) add significant flux. Above $6500 \AA$, line emission quickly gets stronger. This is due both to the increasing number and strength of emission lines. Above $8600 \AA$ the averaged flux from emission lines is a factor of 6 higher than the continuum emission. These numbers become relevant for measurements with spectrographs with lower resolution.

Even if their average value is negligible in all but the redmost optical spectral ranges, fluxes of sky emission lines become relevant locally. As Table 3 shows, their number may locally be rather high and even, in the near-IR, dominate the whole spectrum.

\subsection{Night-sky emission lines}

In total, positions, widths and peak fluxes for $N=2810$ emission lines have been measured. The strongest are at $10^{3}$ times the sky emission continuum. The UV-blue has many resolved lines from the rotational bands of $\mathrm{O}_{2}$, specifically the Herzberg I and Chamberlain bands. Their intensity is up to 10 times the continuum value. They appear all the way down to our spectral limit, about $3140 \AA$ A.

The wavelength range $4000-5500 \AA$ is sparsely populated. The red spectra are dominated by $\mathrm{OH}$ (Meinel) lines, with some regions with strong telluric $\mathrm{O}_{2}$ absorption.

\section{Conclusions}

This paper provides the flux-calibrated optical night-sky emission spectrum for the wavelength range 3140-10400 $\AA$ measured at high resolution with the echelle spectrograph UVES. It is based on the deepest high-resolution exposures of the 
night-sky emission taken so far. Faint continuum sky emission has a level of $1-2 \times 10^{-17} \mathrm{cgs}$. A total of 2810 night-sky emission lines has been carefully selected and measured, with an average accuracy of $17 \mathrm{m \AA}$.

This set of data could become useful to serve as a template for the subtraction of sky emission lines and for wavelength calibration. The night-sky emission flux can be used as an input for exposure time calculators and for planning sky-limited observations.

Figures 7-41 are published in electronic form ${ }^{3}$. Tables 4-9, along with the 1D-spectra as FITS files, are available in electronic form ${ }^{4}$. There is also a web site for rapid browsing ${ }^{5}$.

ESO is interested in collaborations for an ongoing analysis of the data material presented here.

Acknowledgements. RWH would like to thank Jean-Gabriel Cuby from Paranal Science Operations for initiating this project, Sandro D'Odorico for many useful suggestions and Nando Patat for stimulating discussions about extinction correction.

\section{References}

Abrams, M. C., Davis, S. P., Rao, M. L. P., Engleman, R., \& Brault, J. W. 1994, ApJS, 93, 351

Ballester, P., Modigliani, A., Boitquin, O., et al. 2000, The ESO Messenger, 101, 31

Dekker, H., D’Odorico, S., Kaufer, A., Delabre, B., \& Kotzlowski, H. 2000, in Optical and IR telescope instrumentation and detectors, Proc. SPIE, 4008, 534

Edlén, B. 1966, Metrologia, 2, 71

Garstang, R. H. 1989, PASP, 101, 306

Leinert, C., Bowyer, S. Haikala, L. K., et al. 1998, A\&AS, 127, 1

Lidman, C., Cuby, J.-G., \& Rousselot, P. 2000, http://www. eso.org/instruments/isaac/oh/

Osterbrock, D. E., Fulbright, J. P., Martel, A. R., Keane, M. J., \& Trager, S. C. 1996, PASP, 108, 277

Osterbrock, D. E., Fulbright, J. P., \& Bida, T. A. 1997, PASP, 109, 614

Patat, F. 2003, A\&A, 400, 1183

Roach, F. E., \& Meinel, 1955, ApJ, 122, 544

Rousselot, P., Lidman, C., Cuby, J.-G., Moreels, G., \& Monnet, G. 2000, A\&A, 354, 1134

Tüg, H. 1980, A\&A, 82, 195

\footnotetext{
${ }^{3}$ http://www.edpsciences.org

${ }^{4}$ via anonymous ftp to cdsarc.u-strasbg. fr (130.79.128.5)

or via

http://cdsweb.u-strasbg.fr/cgi-bin/qcat? J/A+A/407/1157

${ }^{5}$ http://www.eso.org/qc/UVES/pipeline/

sky_spectrum.html
} 
R. W. Hanuschik: Night-sky emission flux, Online Material p 1

\section{Online Material}




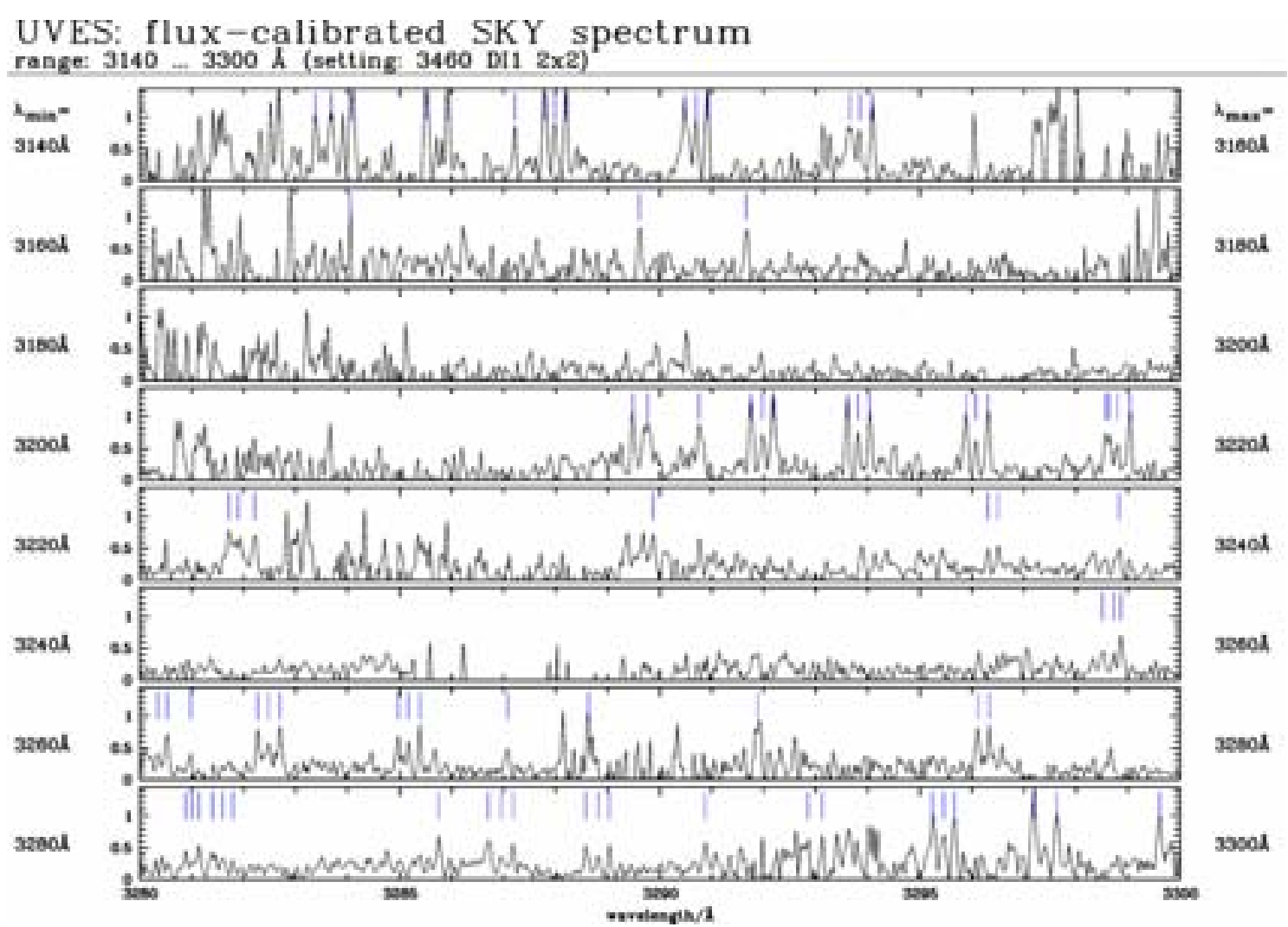

Fig. 7. The night-sky spectrum as seen by UVES, covering the wavelength range $\lambda \lambda 3140-3300 \AA$. The vertical bars mark line measurements from this atlas.

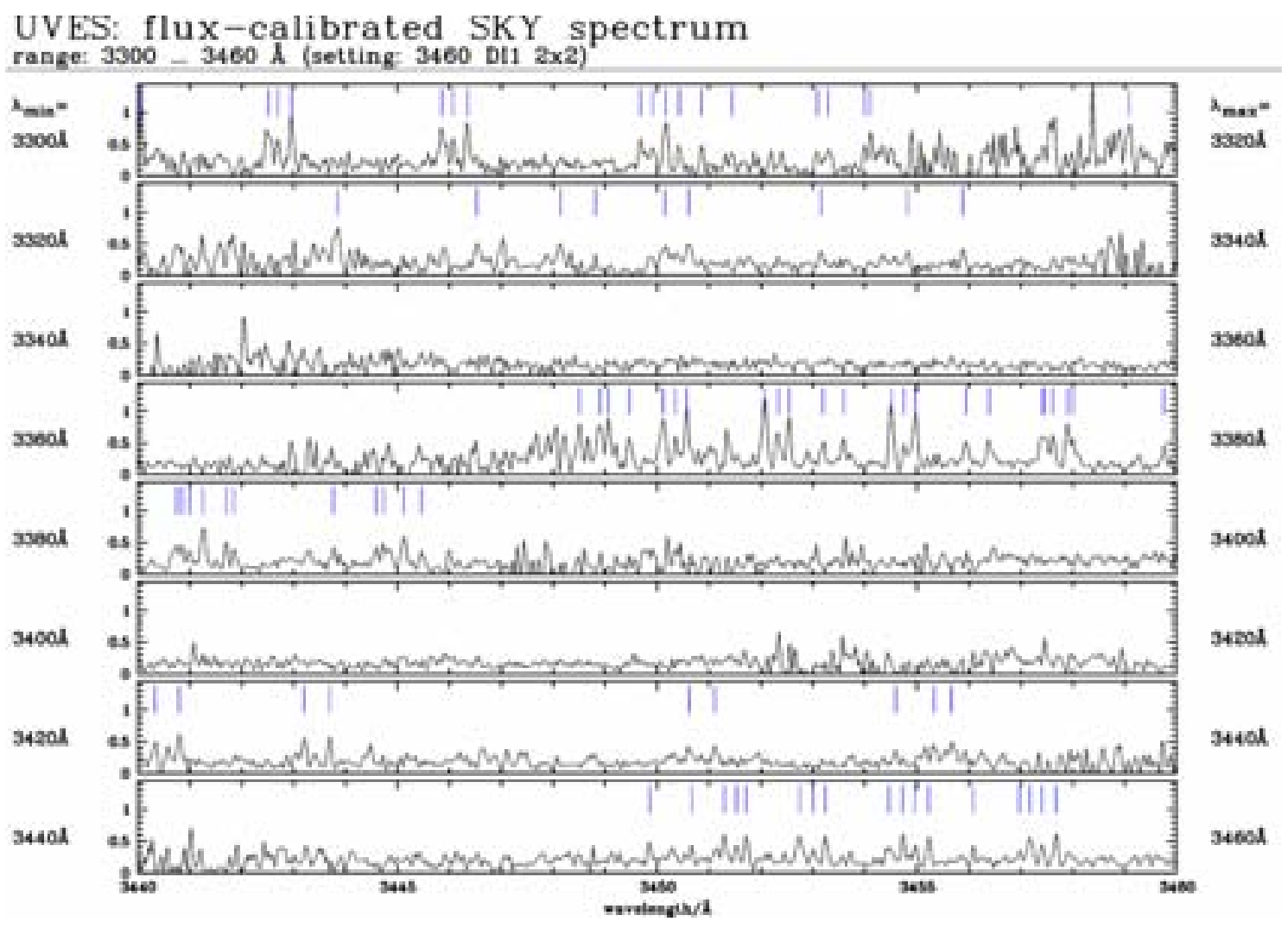

Fig. 8. Same as Fig. 7, for range 3300-3460 ̊. 


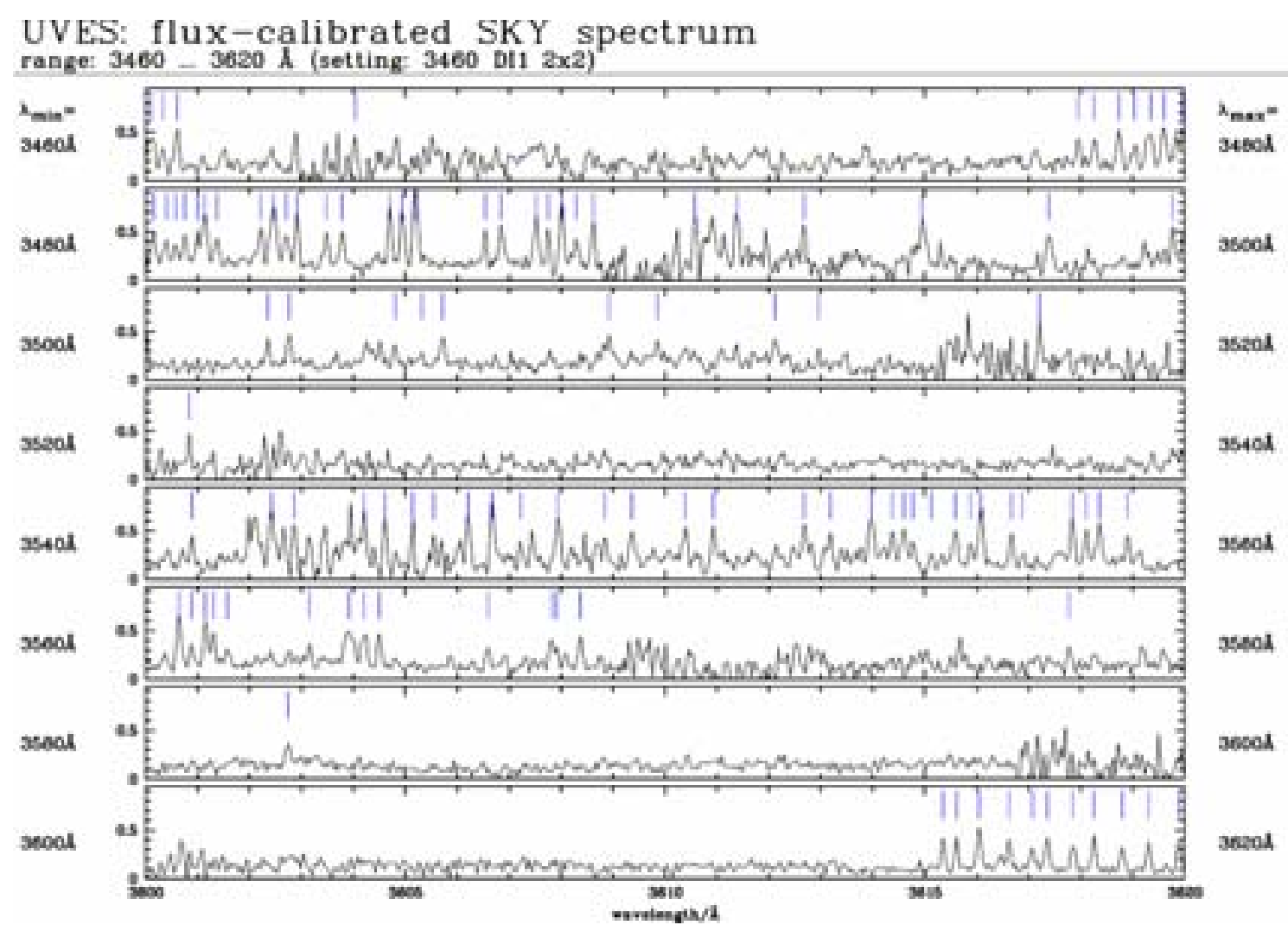

Fig. 9. Same as Fig. 7, for range 3460-3620 Å.

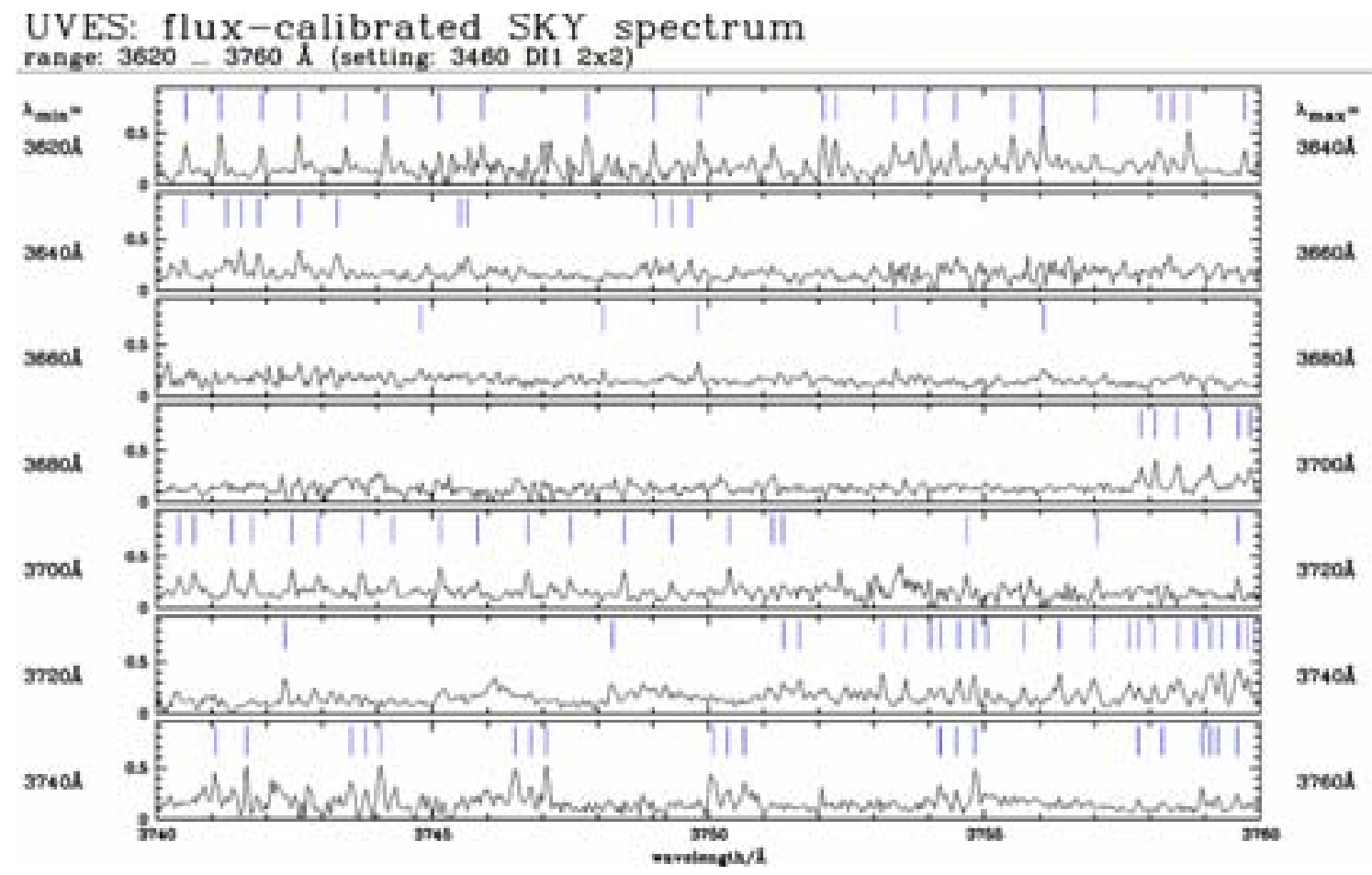

Fig. 10. Same as Fig. 7, for range 3620-3760 A. 


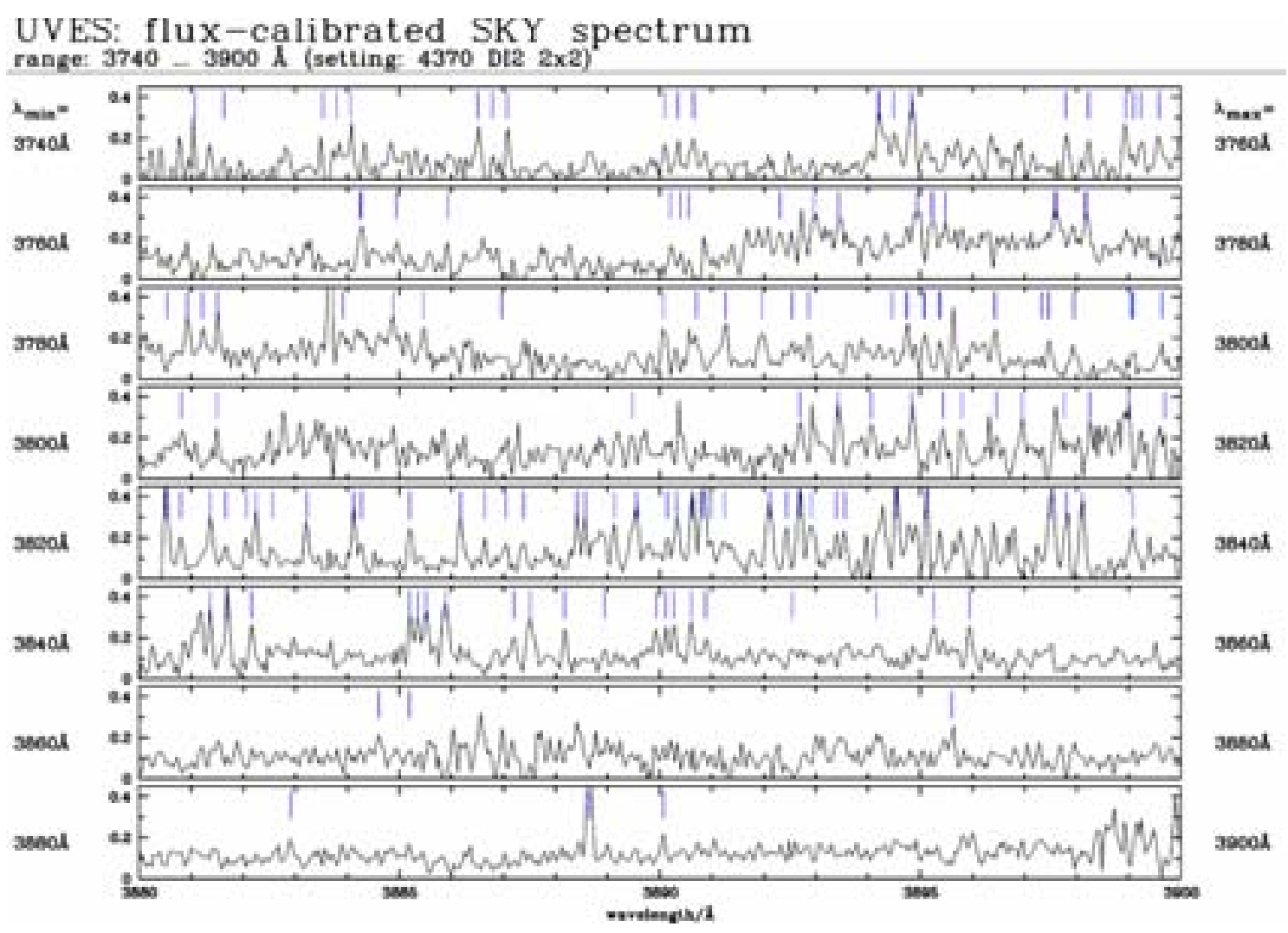

Fig. 11. UVES night-sky spectrum, covering 3740-3900 A. The vertical bars mark line measurements from this atlas. See the text of the Appendix for more general information about these plots.

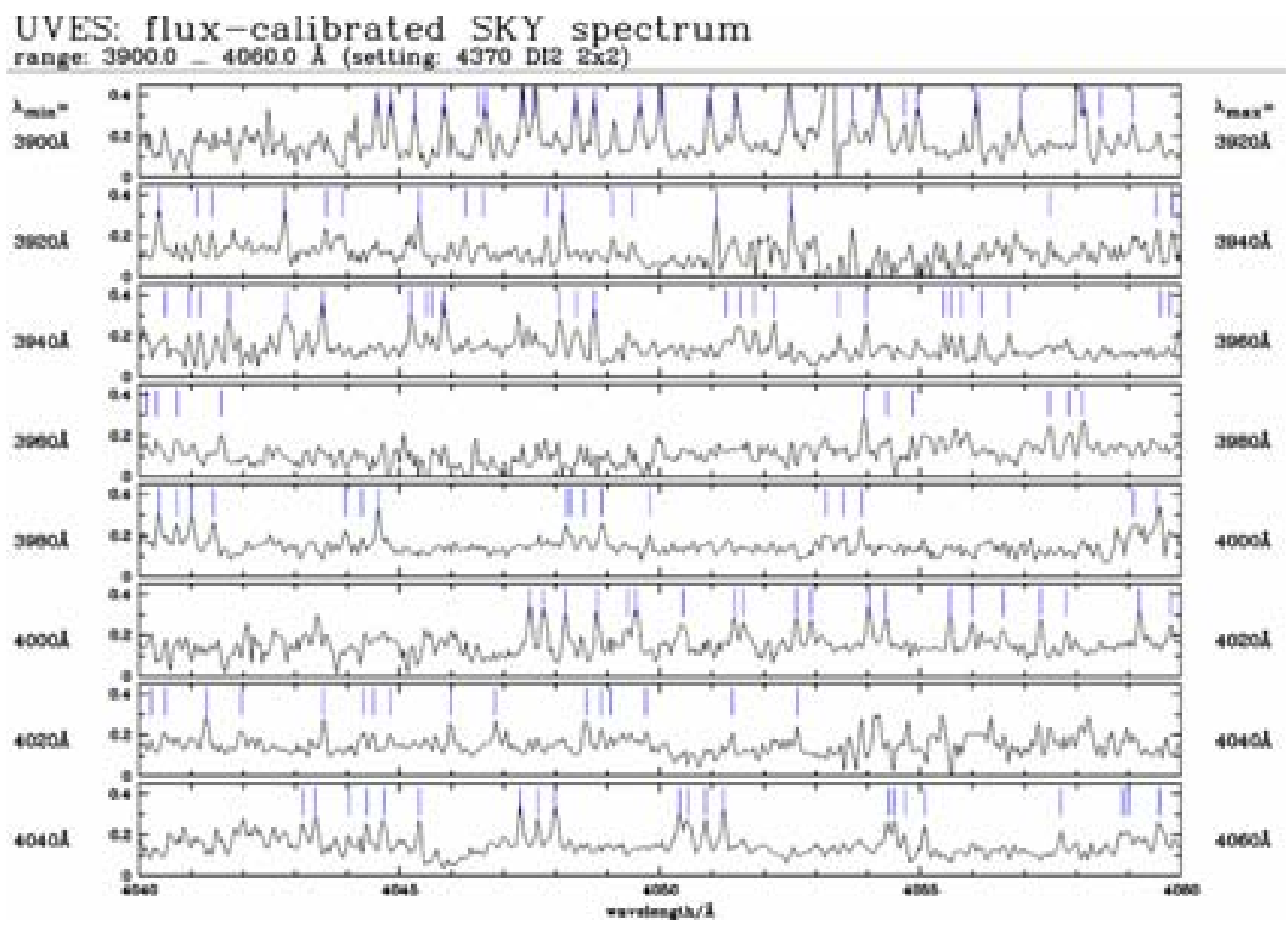

Fig. 12. Same as Fig. 11, for range 3900-4060 A. 
UVES: flux-calibrated SKY spectrum

range: $4000-4220 A$ (setting 4370 DH2 $2 \times 2$ )

(1001

Fig. 13. Same as Fig. 11, for range 4060-4220 Å.

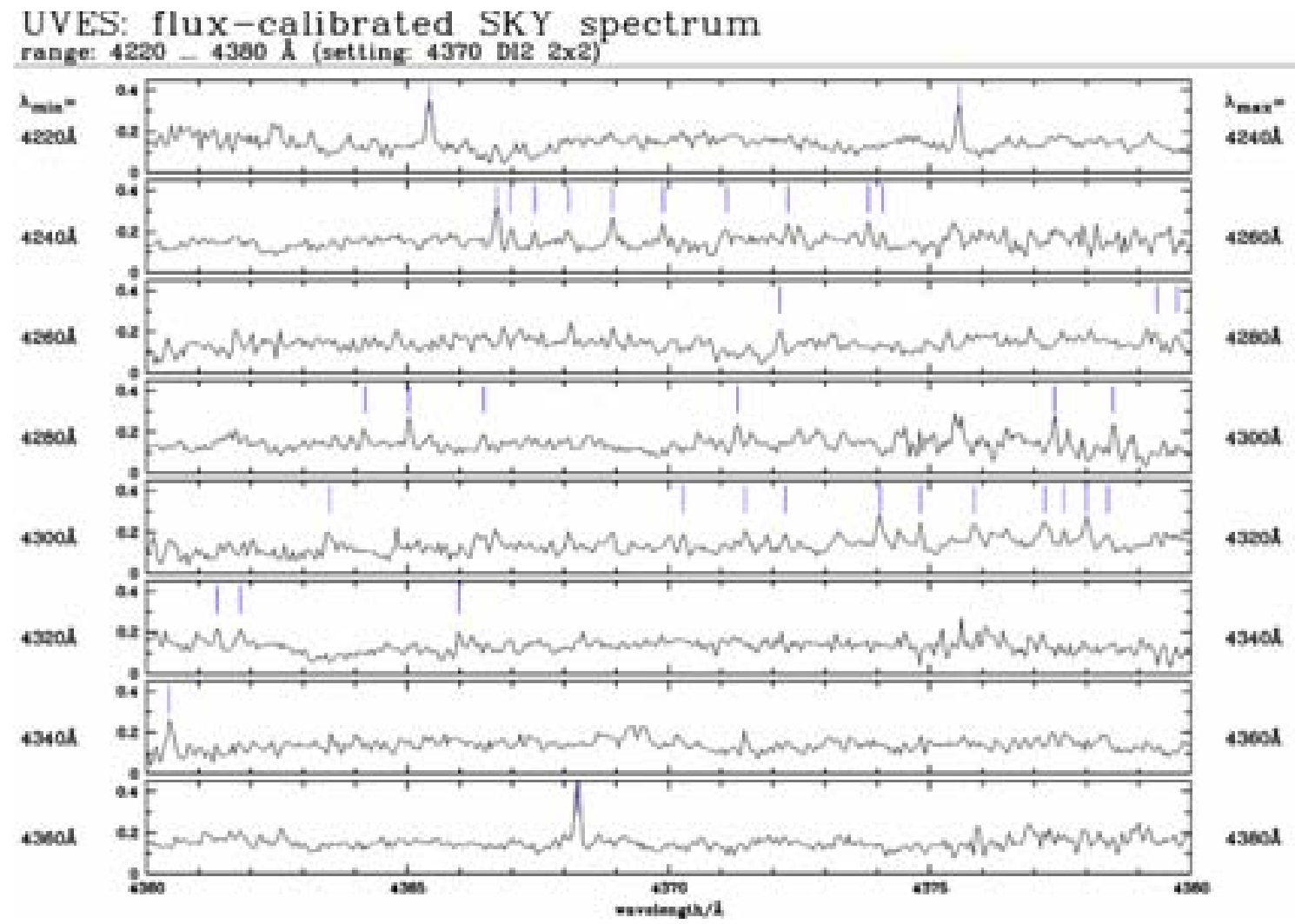

Fig. 14. Same as Fig. 11, for range 4220-4380 ̊. 


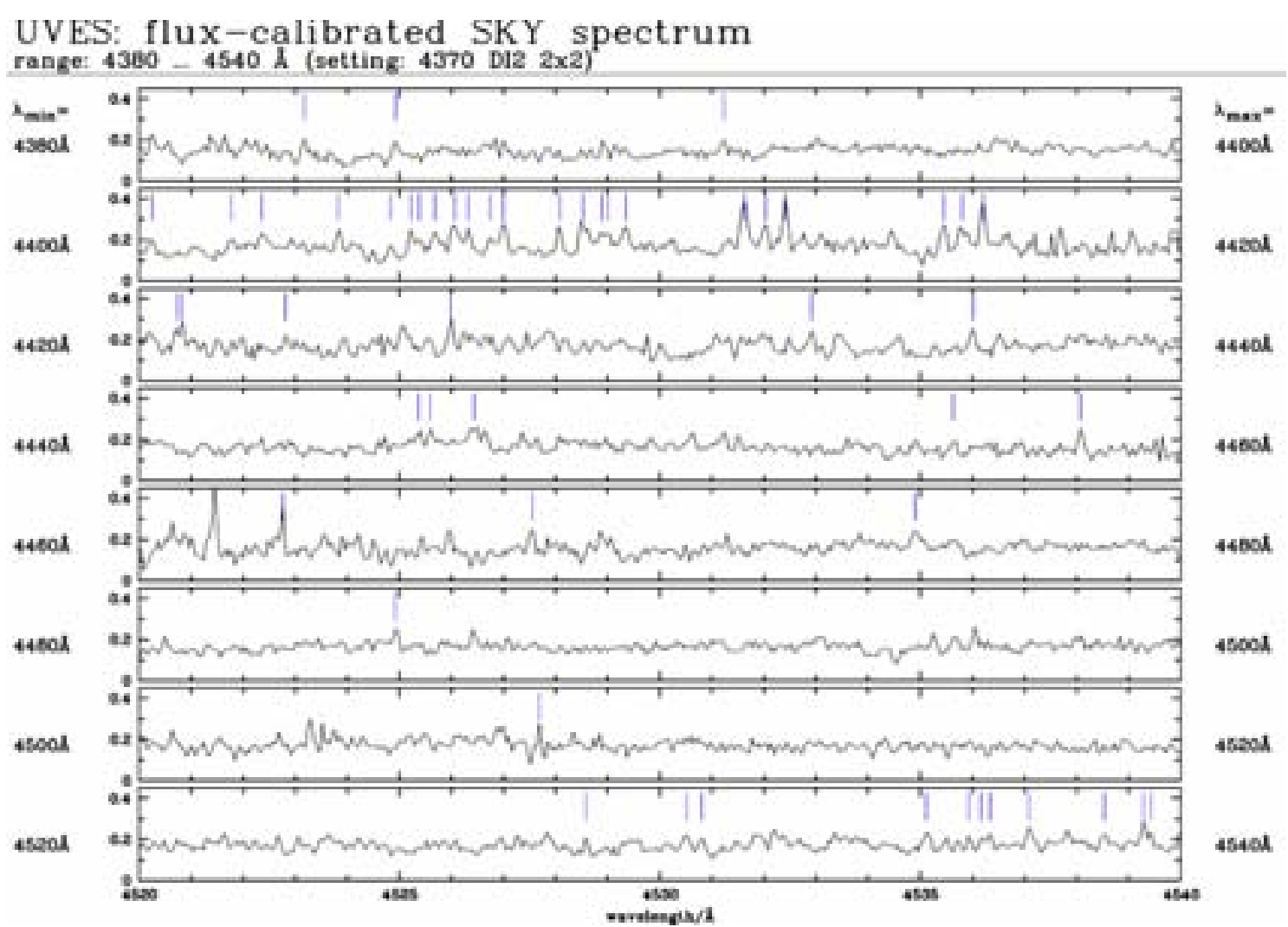

Fig. 15. Same as Fig. 11, for range 4380-4540 Å.

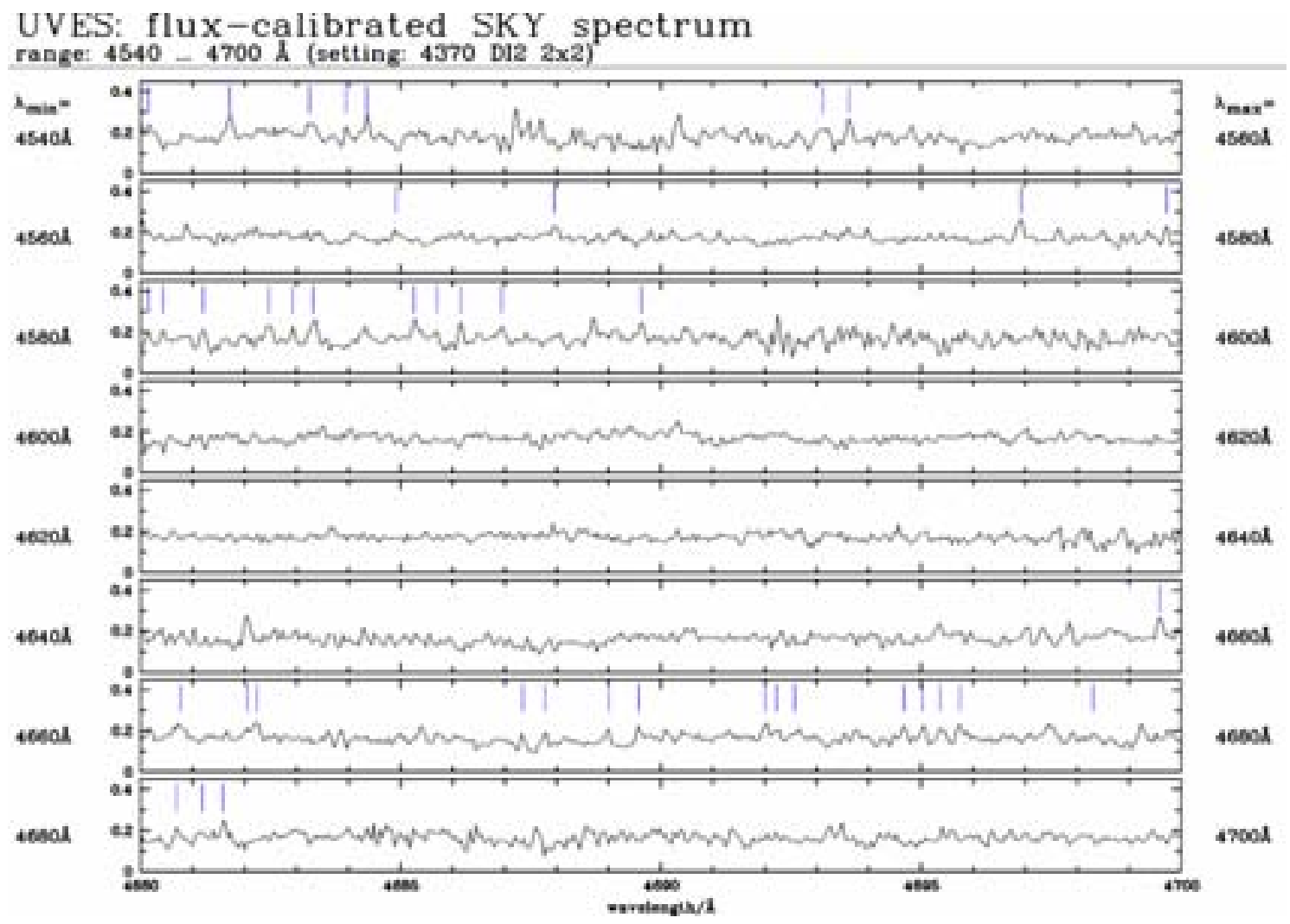

Fig. 16. Same as Fig. 11, for range 4540-4700 Å. 


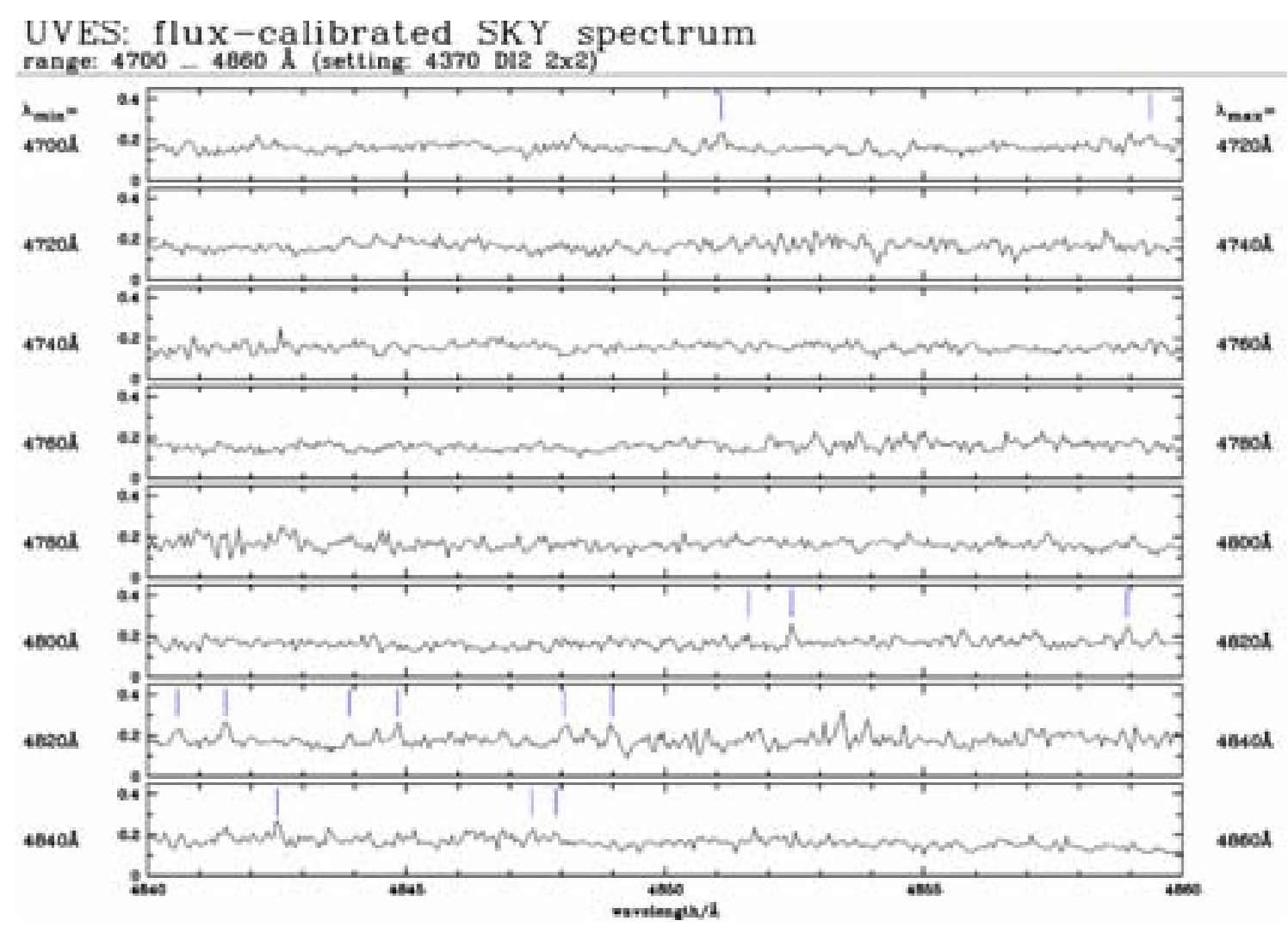

Fig. 17. Same as Fig. 11, for range 4700-4860 Å.

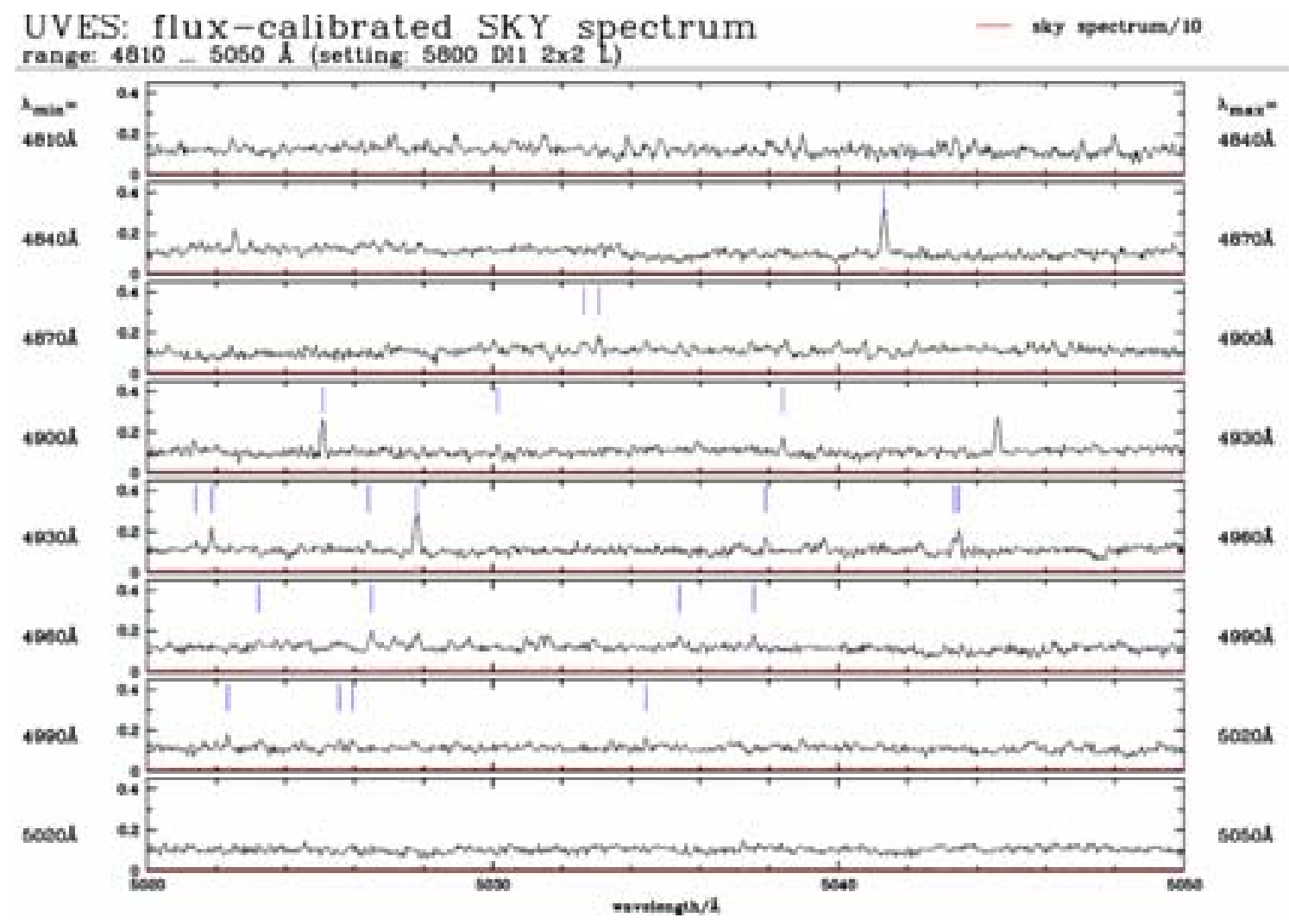

Fig. 18. UVES night-sky spectrum, covering 4810-5050 ̊. See the text of the Appendix for more general information about these plots. The vertical bars mark line measurements from this atlas. The sky spectrum is also plotted on a scale compressed by a factor 10 to better show stronger lines. The full circles mark emission lines with identification in the Keck atlas (Osterbrock et al. 1996). 
R. W. Hanuschik: Night-sky emission flux, Online Material p 8

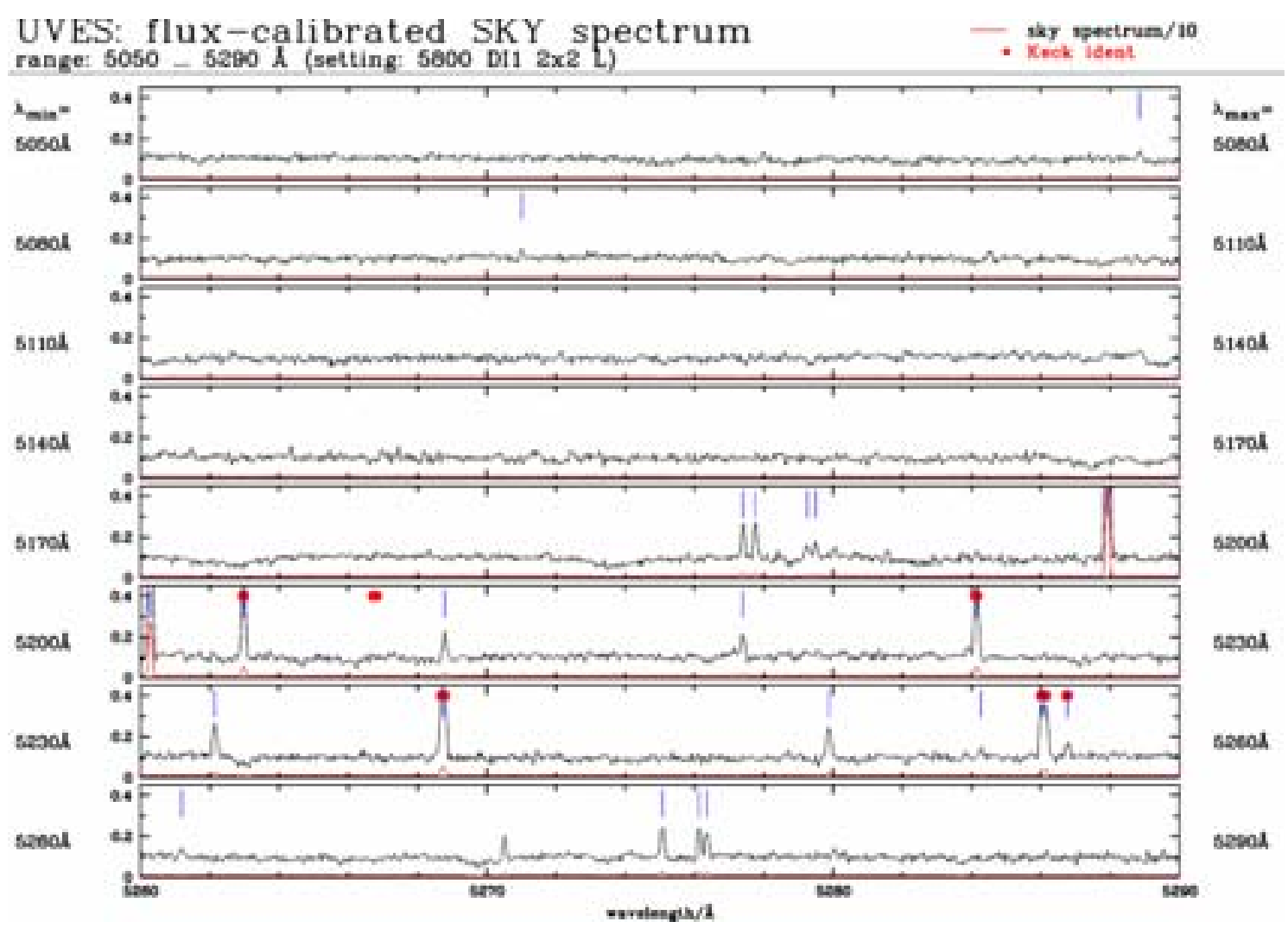

Fig. 19. Same as Fig. 18, for range 5050-5290 А.

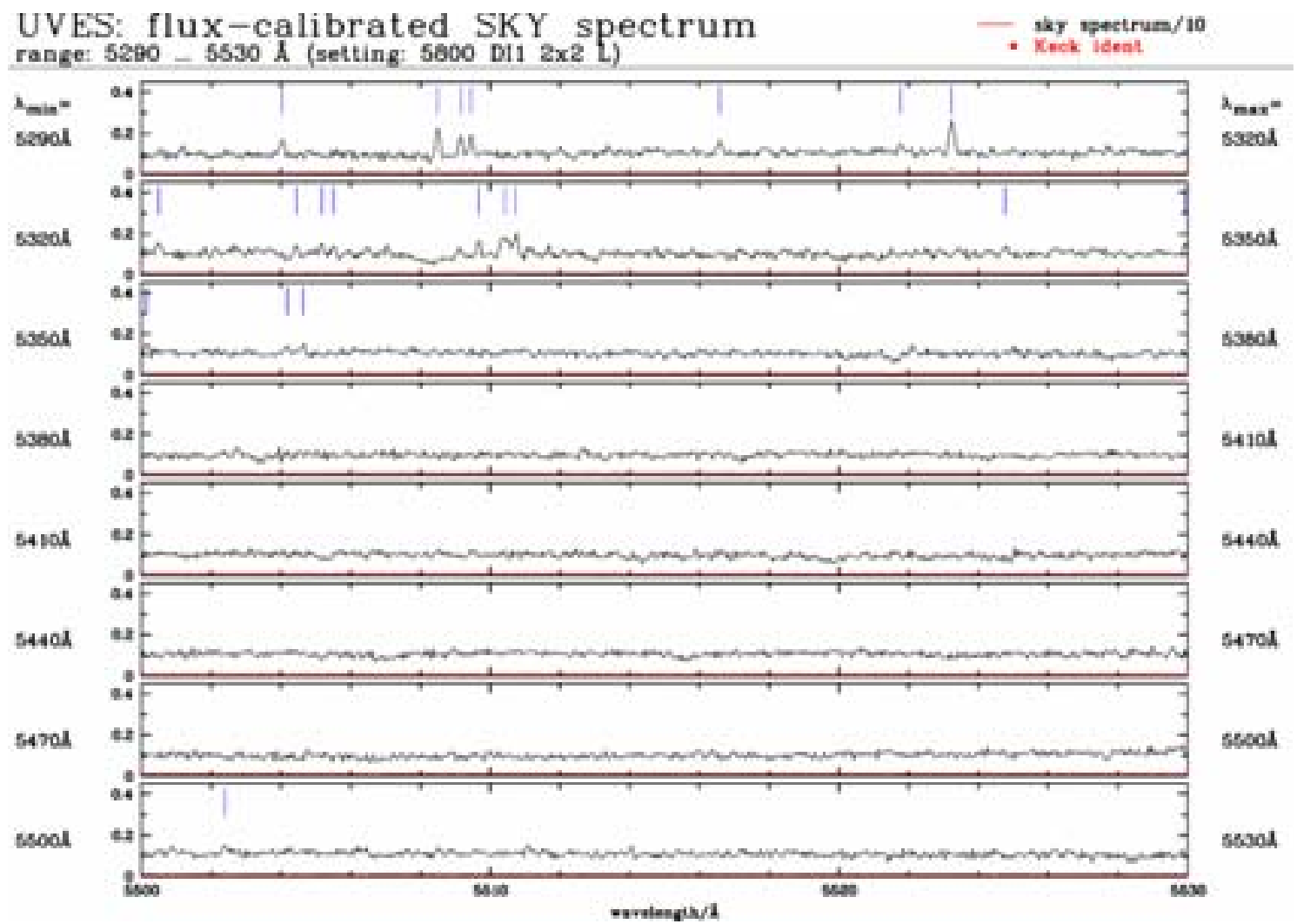

Fig. 20. Same as Fig. 18, for range 5290-5530 A. 
R. W. Hanuschik: Night-sky emission flux, Online Material p 9

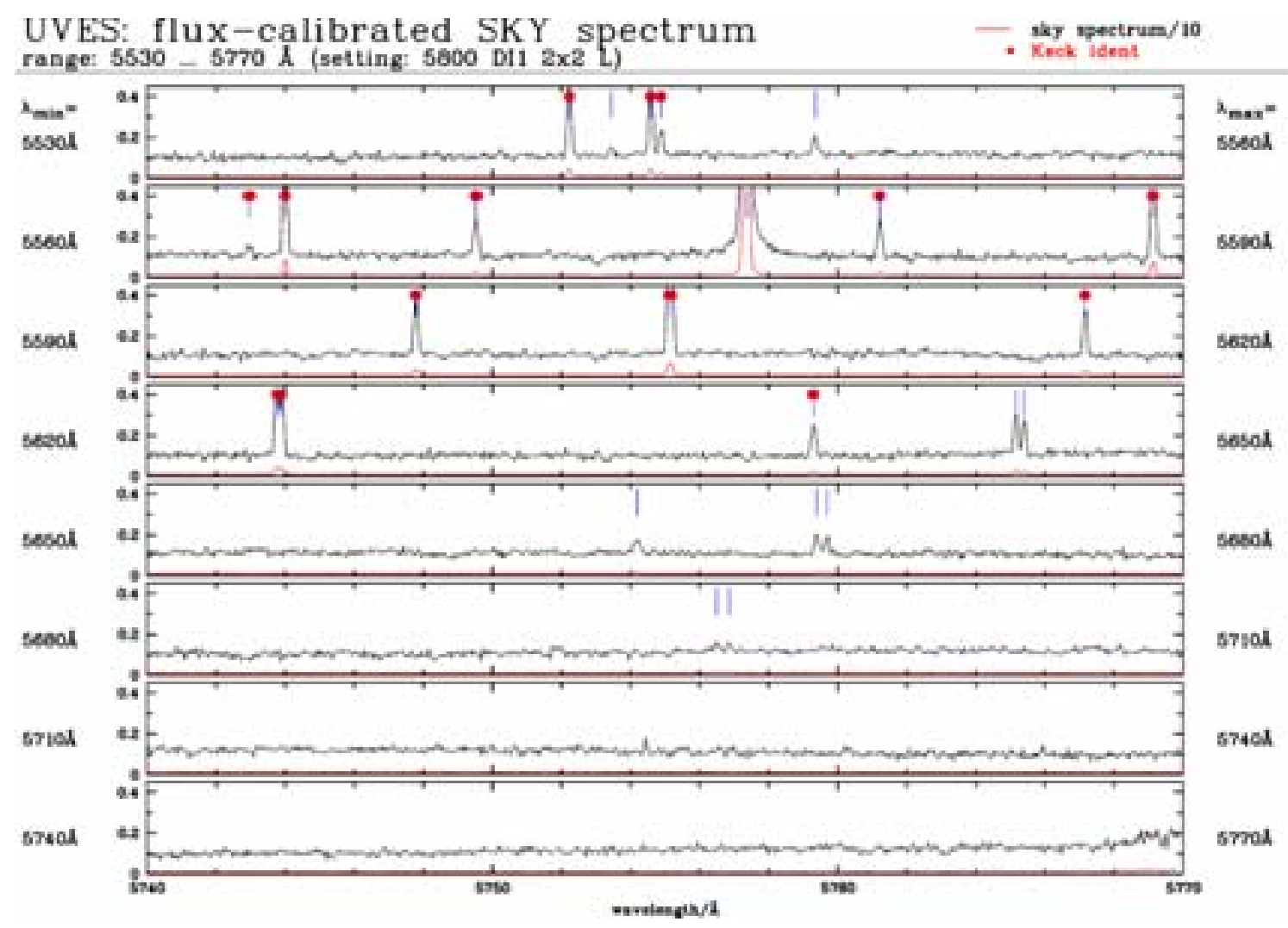

Fig. 21. Same as Fig. 18, for range 5530-5770 ̊̊.

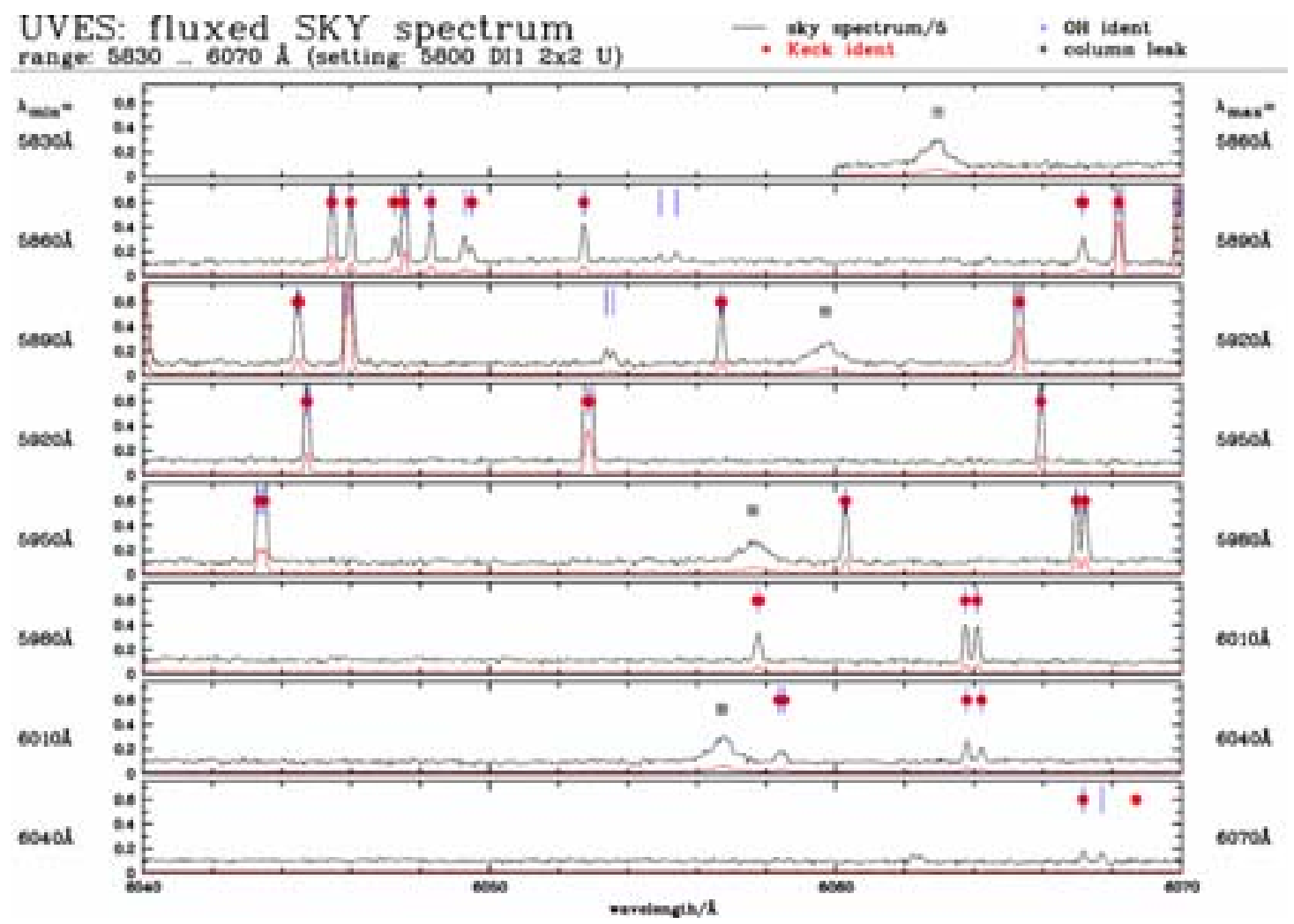

Fig. 22. UVES night-sky spectrum, covering 5830-6070 ̊. See the text of the Appendix for more general information about these plots. The vertical bars mark line measurements from this atlas. The sky spectrum is also plotted on a scale compressed by a factor 5 to better show stronger lines. The full circles mark emission lines with identification in the Keck atlas (Osterbrock et al. 1996). The crosses mark the (aircorrected) positions of identified $\mathrm{OH}$ lines in the Rousselot et al. (2000) and Lidman et al. (2000) lists. Artefacts due to a leaking column are marked by a squared plus. 
R. W. Hanuschik: Night-sky emission flux, Online Material p 10

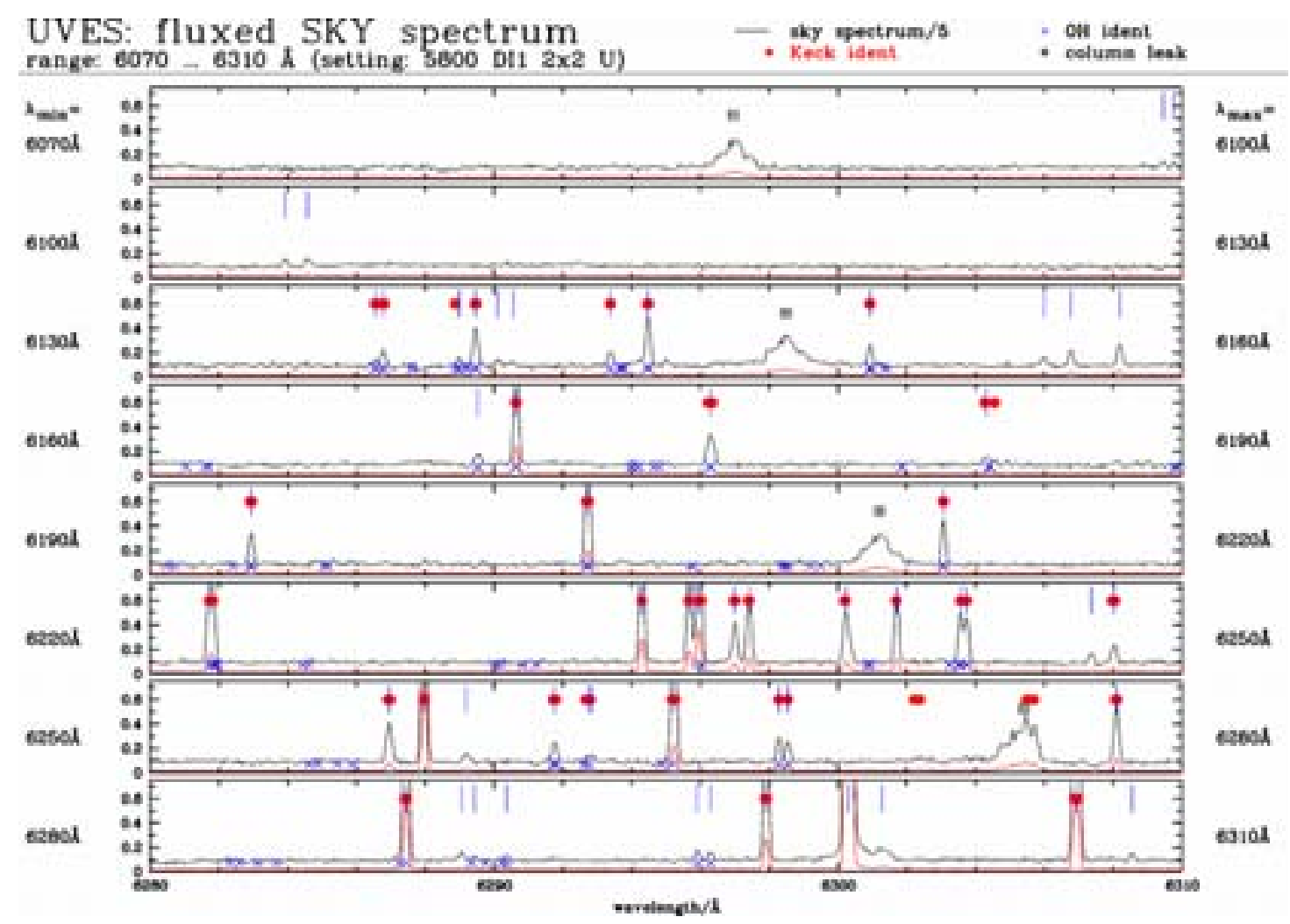

Fig. 23. Same as Fig. 22, for range 6070-6310 А.

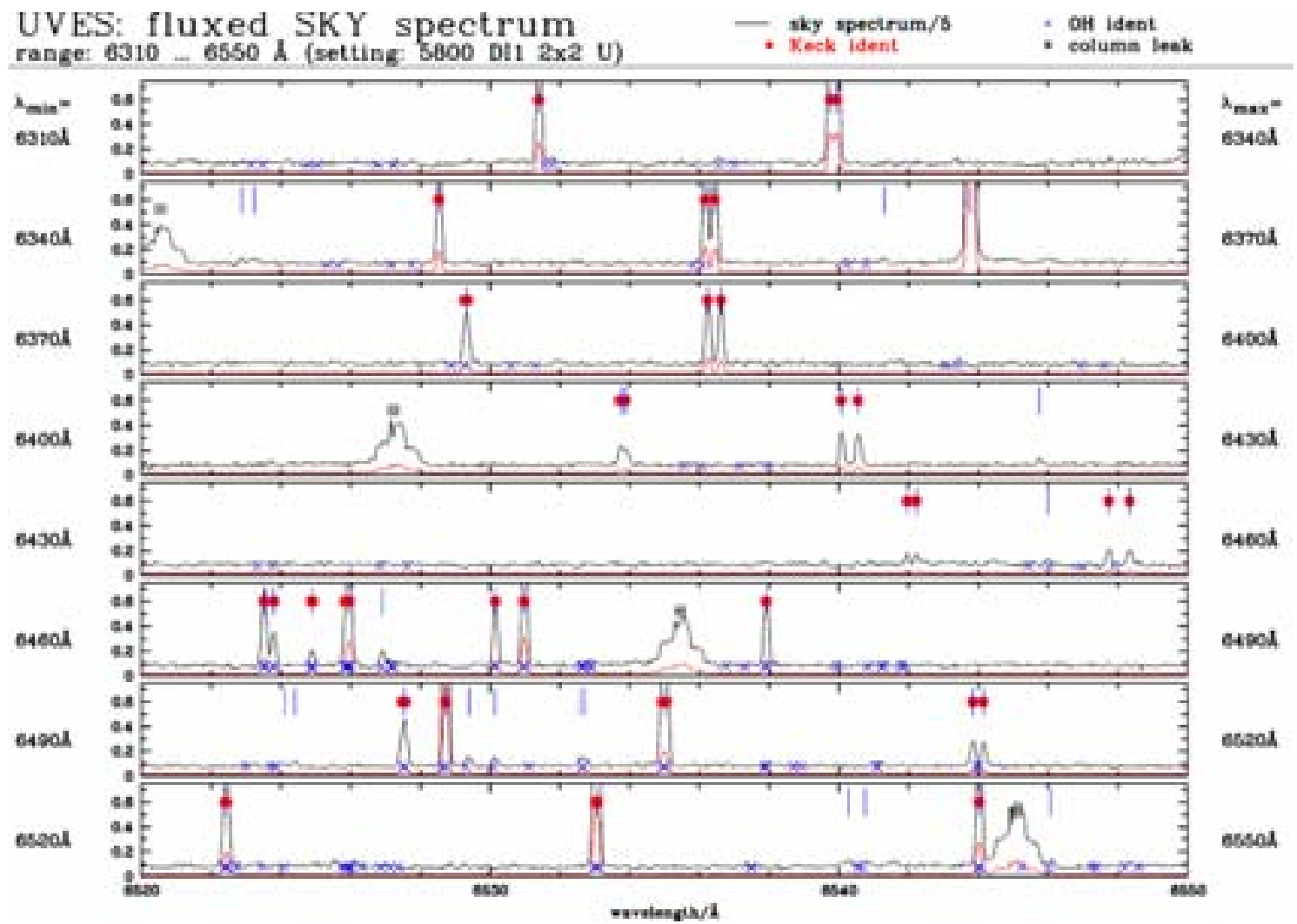

Fig. 24. Same as Fig. 22, for range 6310-6550 A. 
R. W. Hanuschik: Night-sky emission flux, Online Material p 11

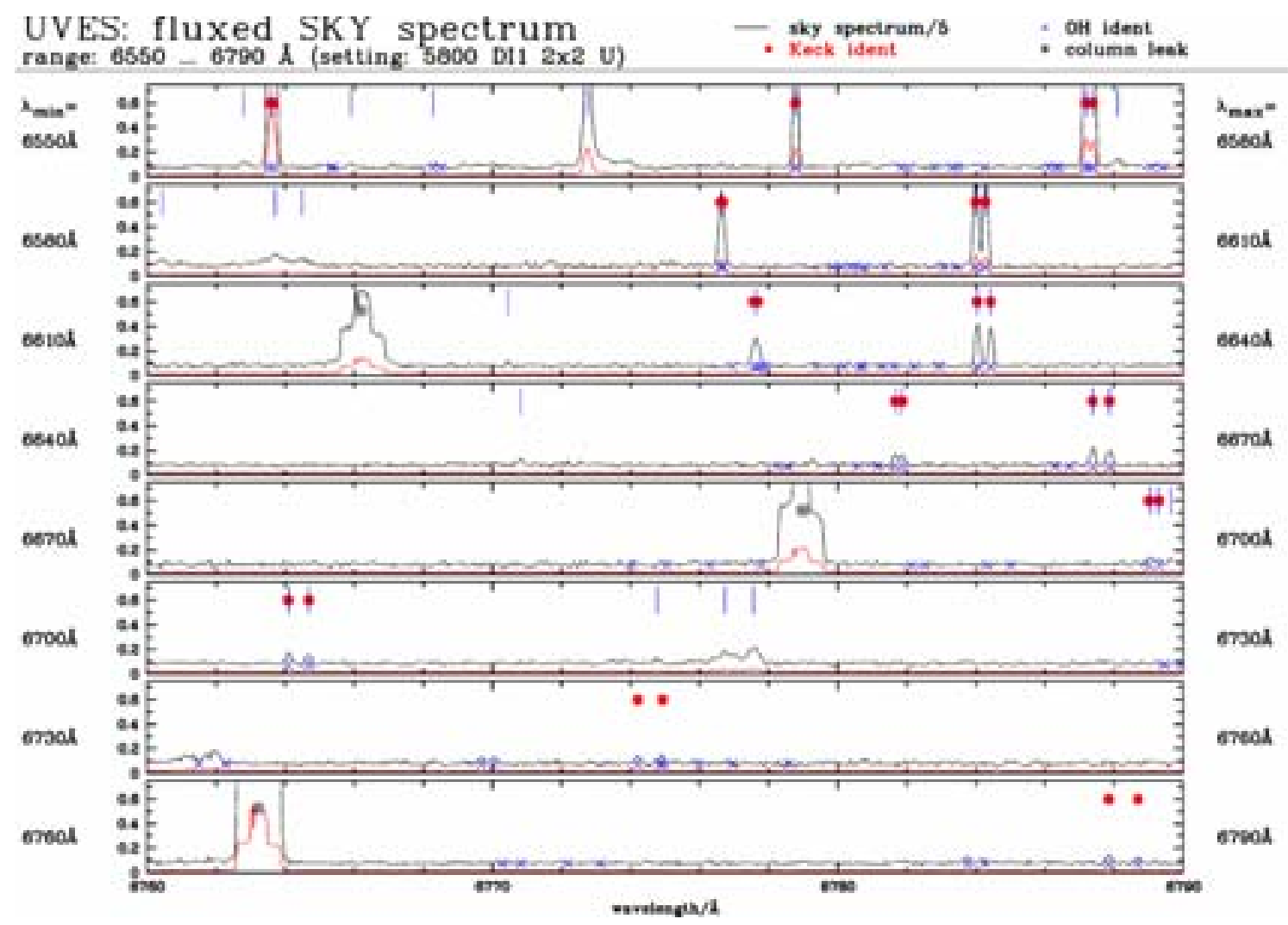

Fig. 25. Same as Fig. 22, for range 6550-6790 ̊̊.

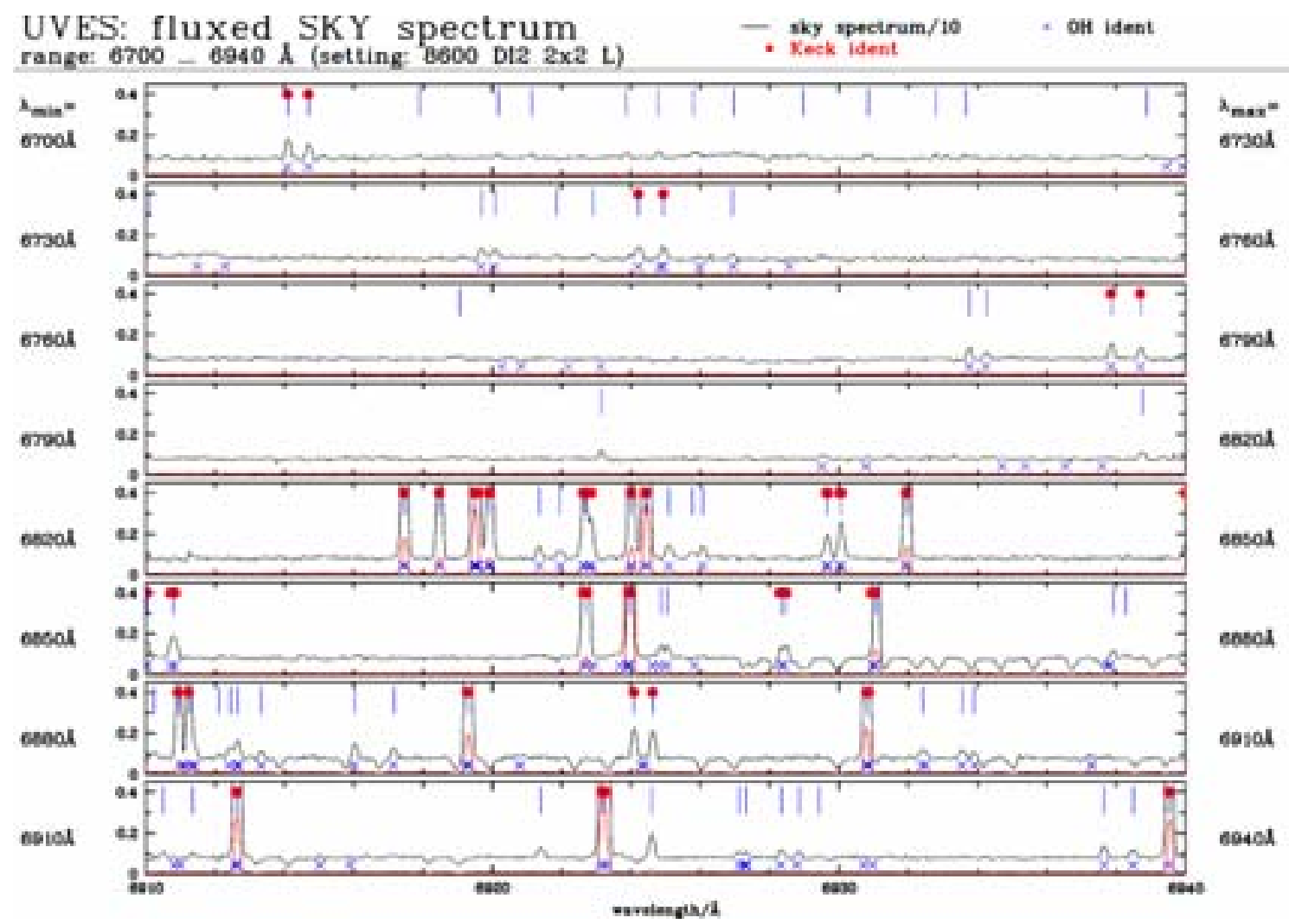

Fig. 26. UVES night-sky spectrum, covering 6700-6940 A. See the text of the Appendix for more general information about these plots. The vertical bars mark line measurements from this atlas. The sky spectrum is also plotted on a scale compressed by a factor of 10 to better show stronger lines. The full circles mark emission lines with identification in the Keck atlas (Osterbrock et al. 1996). The crosses mark the (air-corrected) positions of identified $\mathrm{OH}$ lines in the Rousselot et al. (2000) and Lidman et al. (2000) lists. 
R. W. Hanuschik: Night-sky emission flux, Online Material p 12

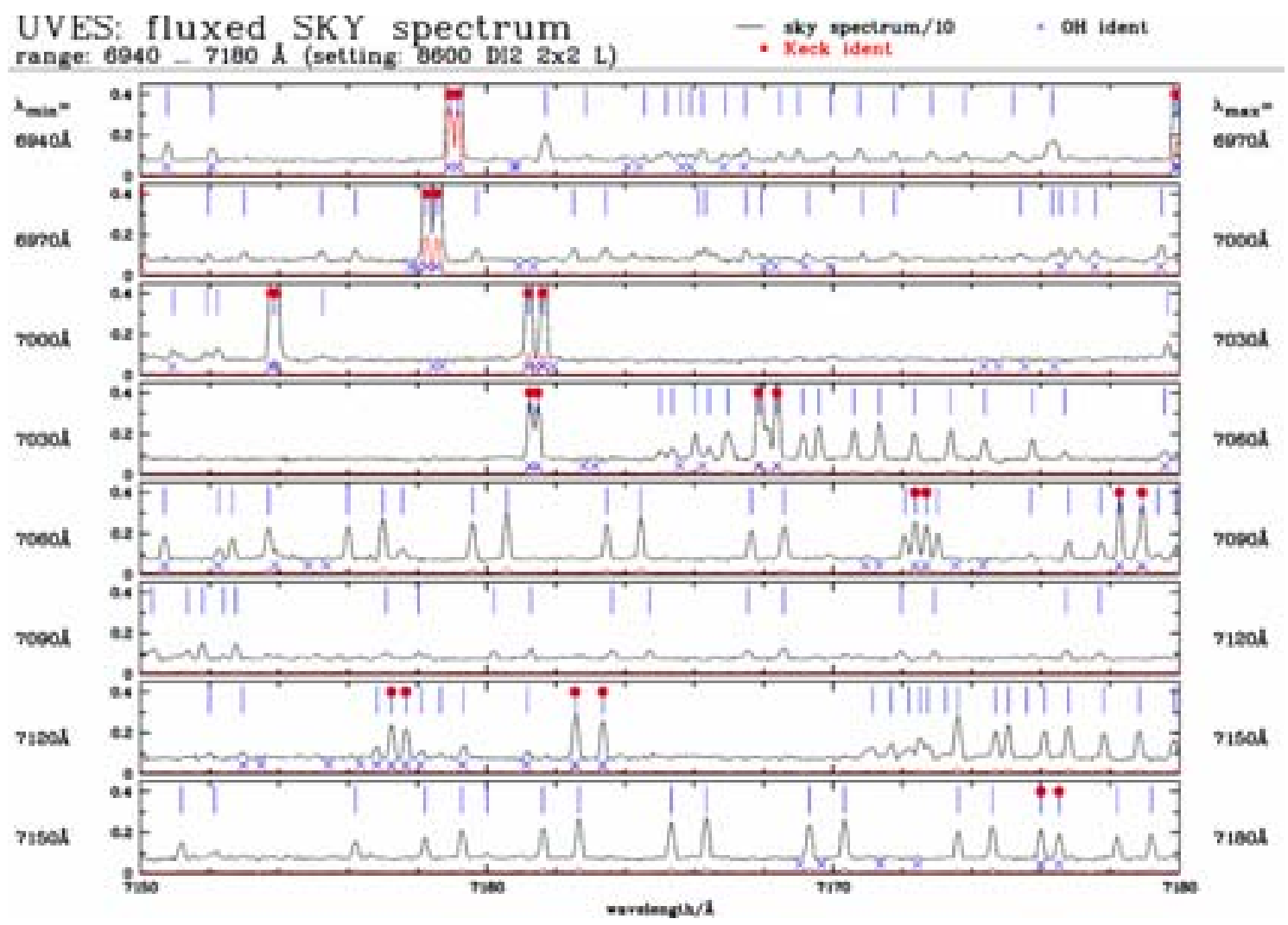

Fig. 27. Same as Fig. 26, for range 6940-7180 Å.

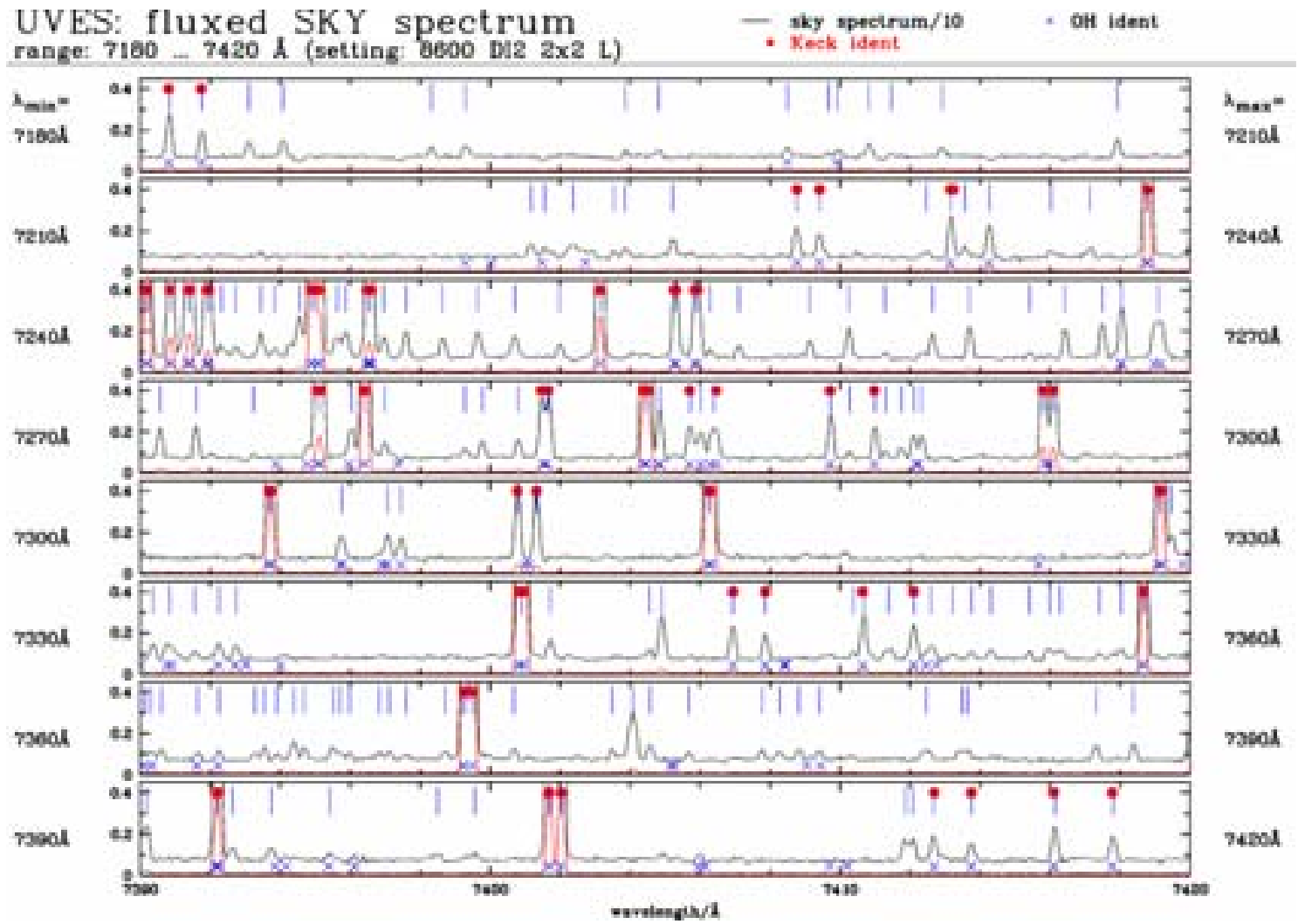

Fig. 28. Same as Fig. 26, for range 7180-7420 A. 
R. W. Hanuschik: Night-sky emission flux, Online Material p 13

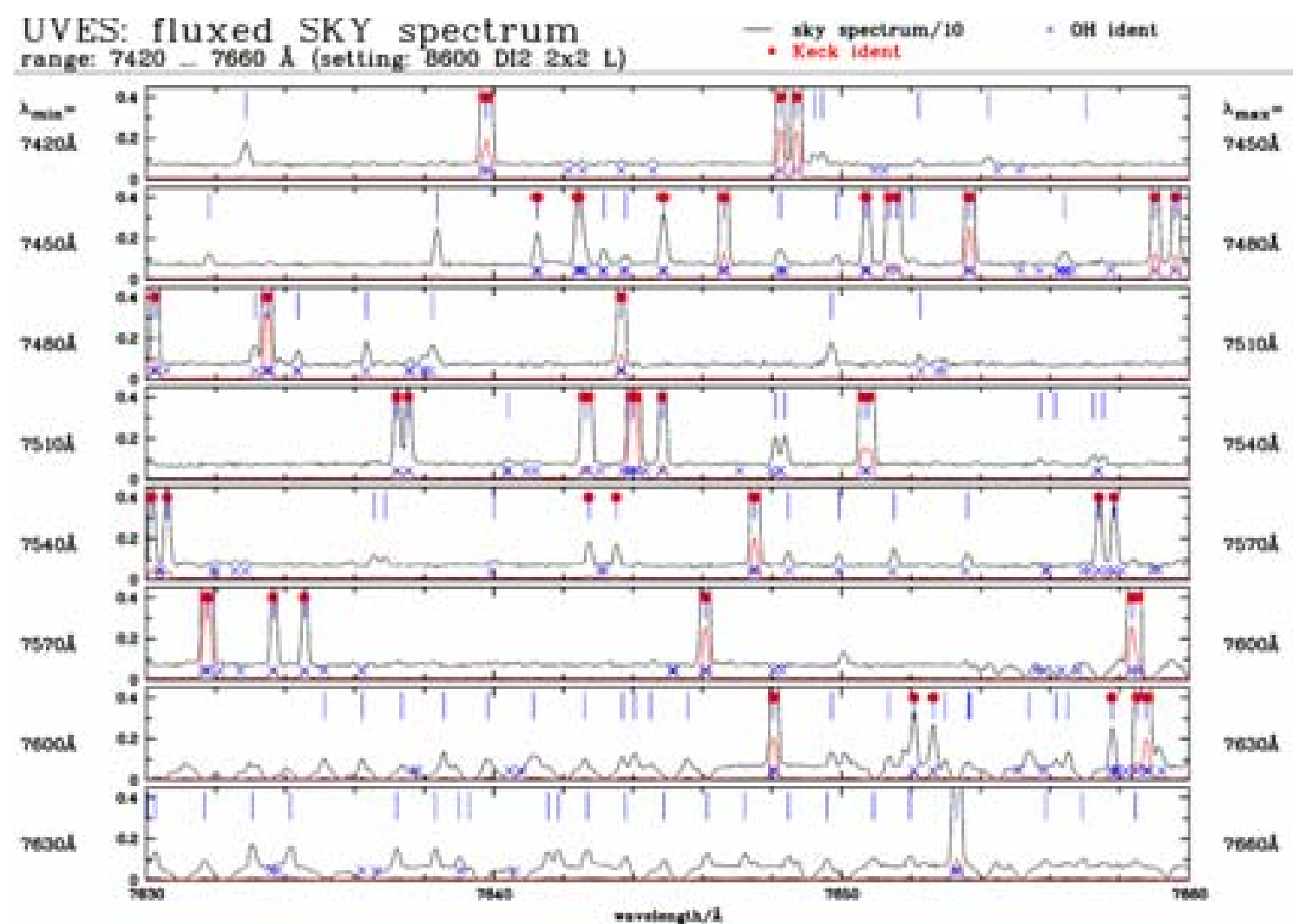

Fig. 29. Same as Fig. 26, for range 7420-7660 A.

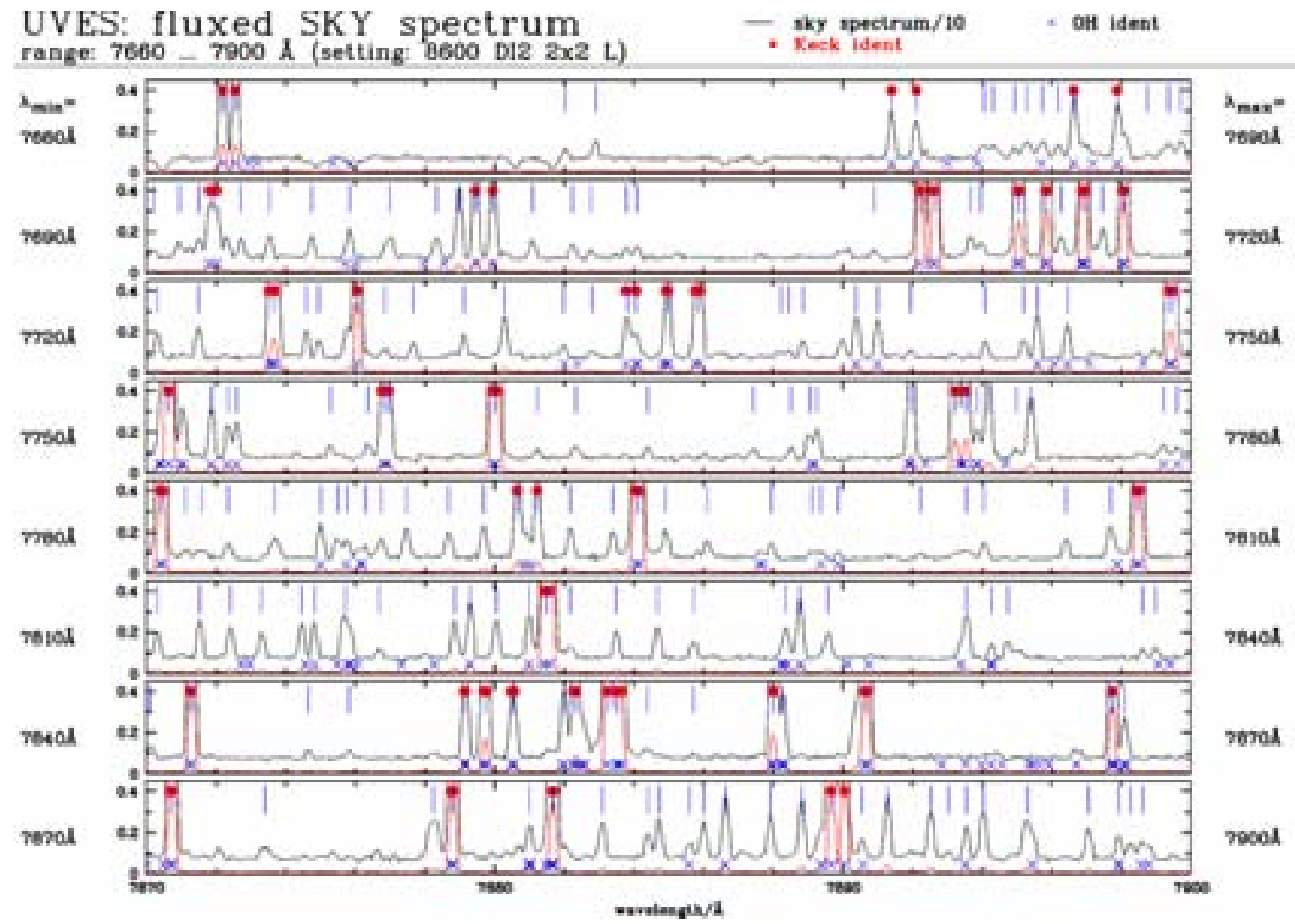

Fig. 30. Same as Fig. 26, for range 7660-7900 ̊. 
R. W. Hanuschik: Night-sky emission flux, Online Material p 14

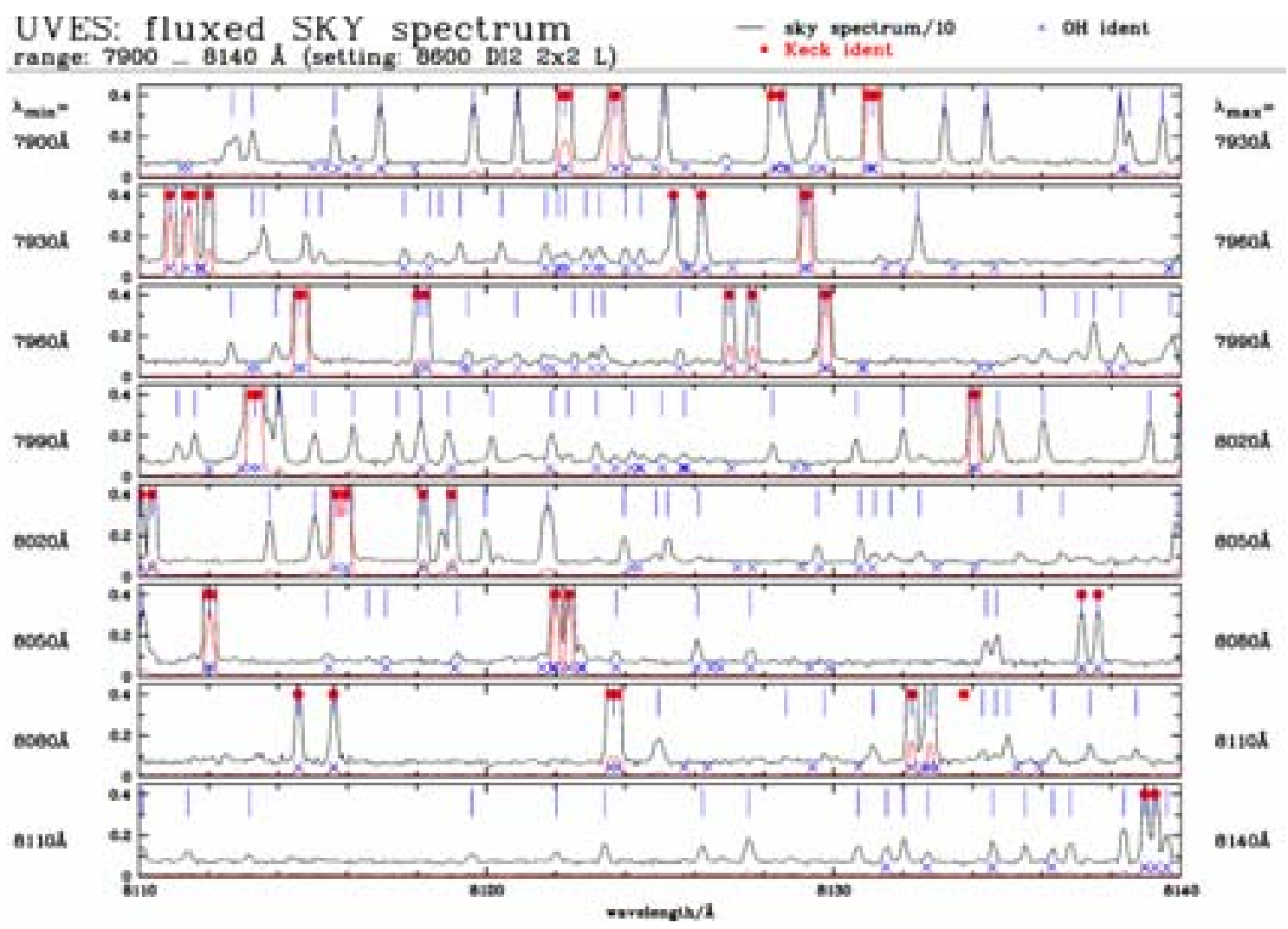

Fig. 31. Same as Fig. 26, for range 7900-8140 Å.

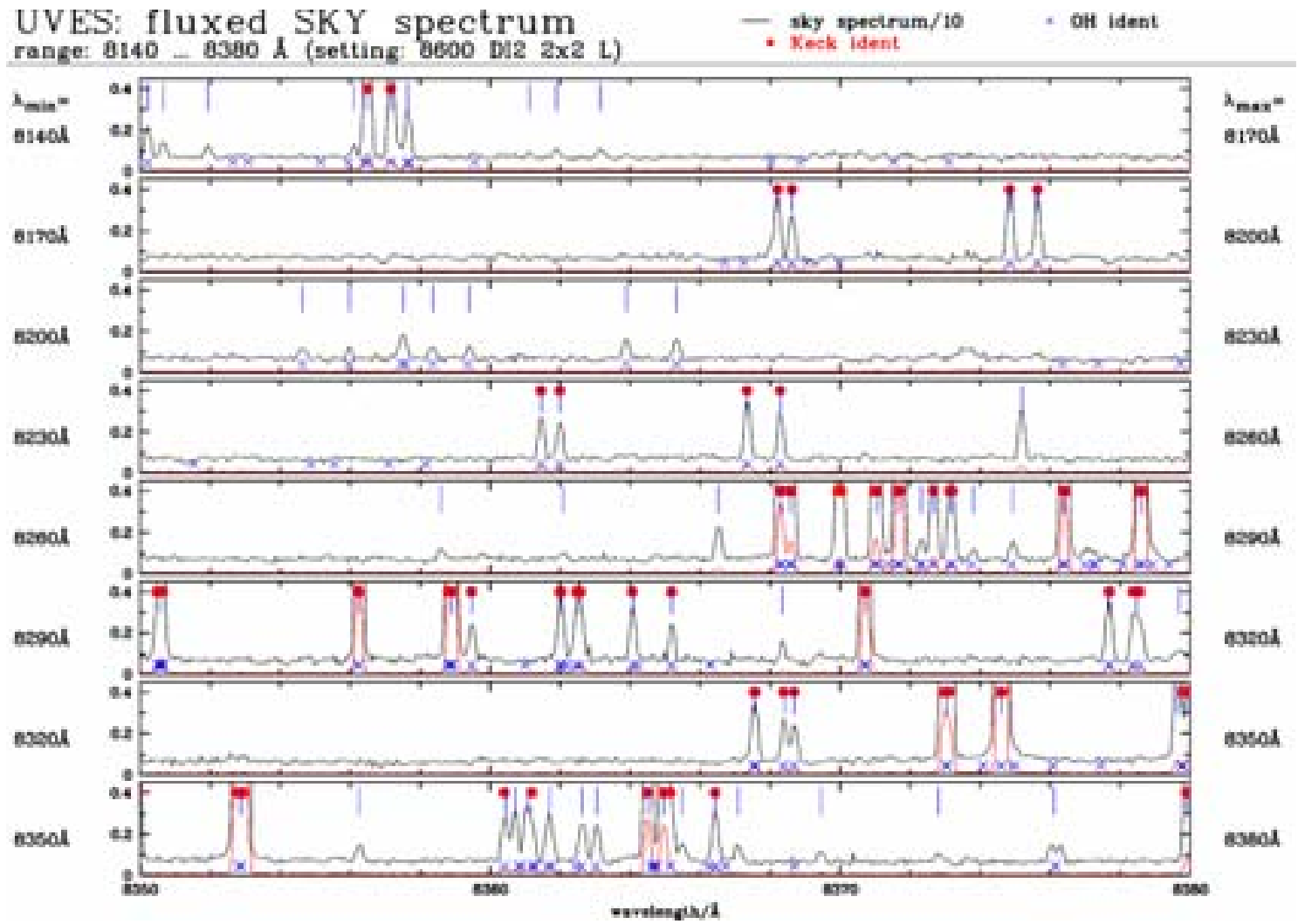

Fig. 32. Same as Fig. 26, for range 8140-8380 A. 
R. W. Hanuschik: Night-sky emission flux, Online Material p 15

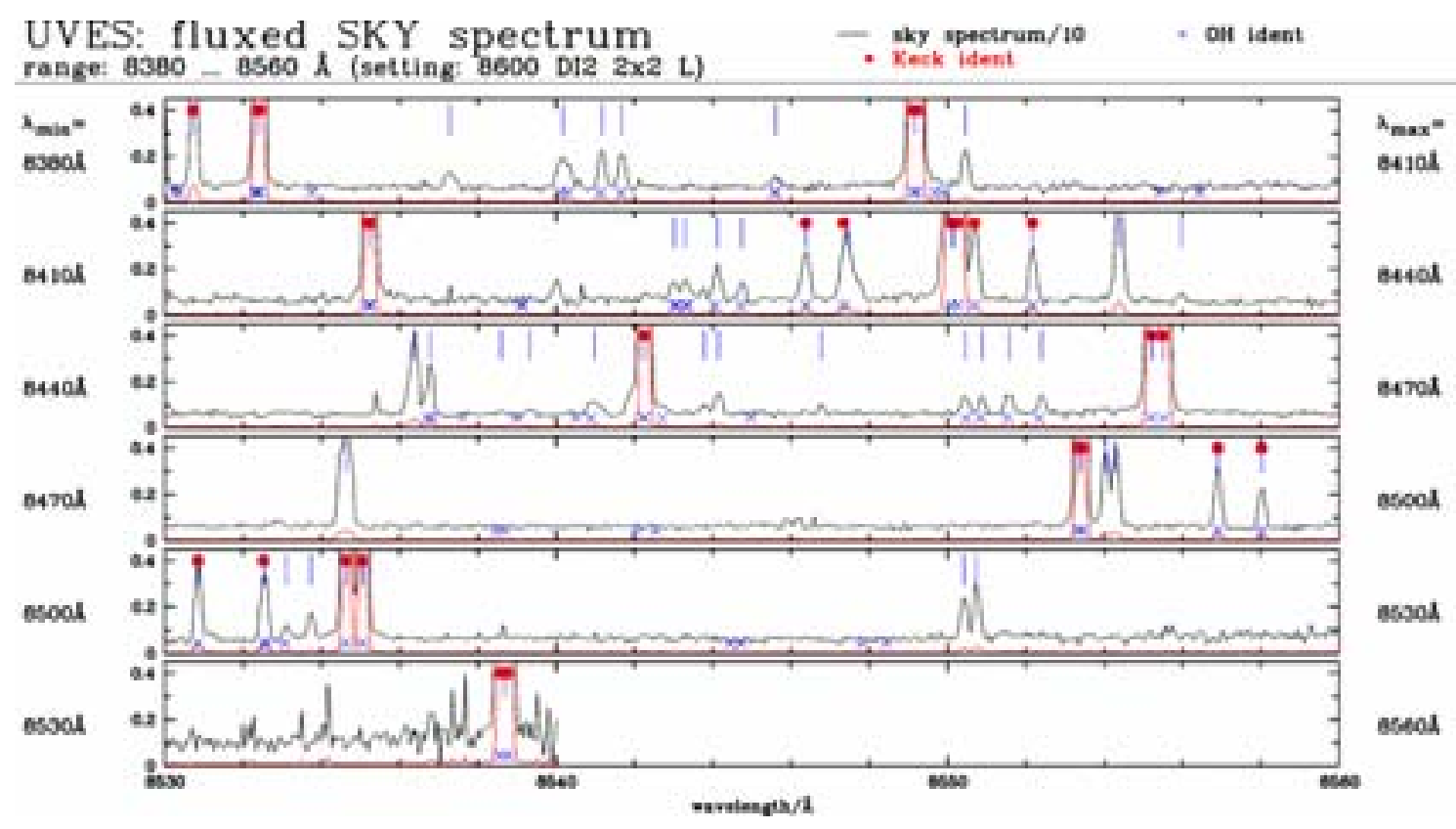

Fig. 33. Same as Fig. 26, for range 8380-8560 ̊̊.

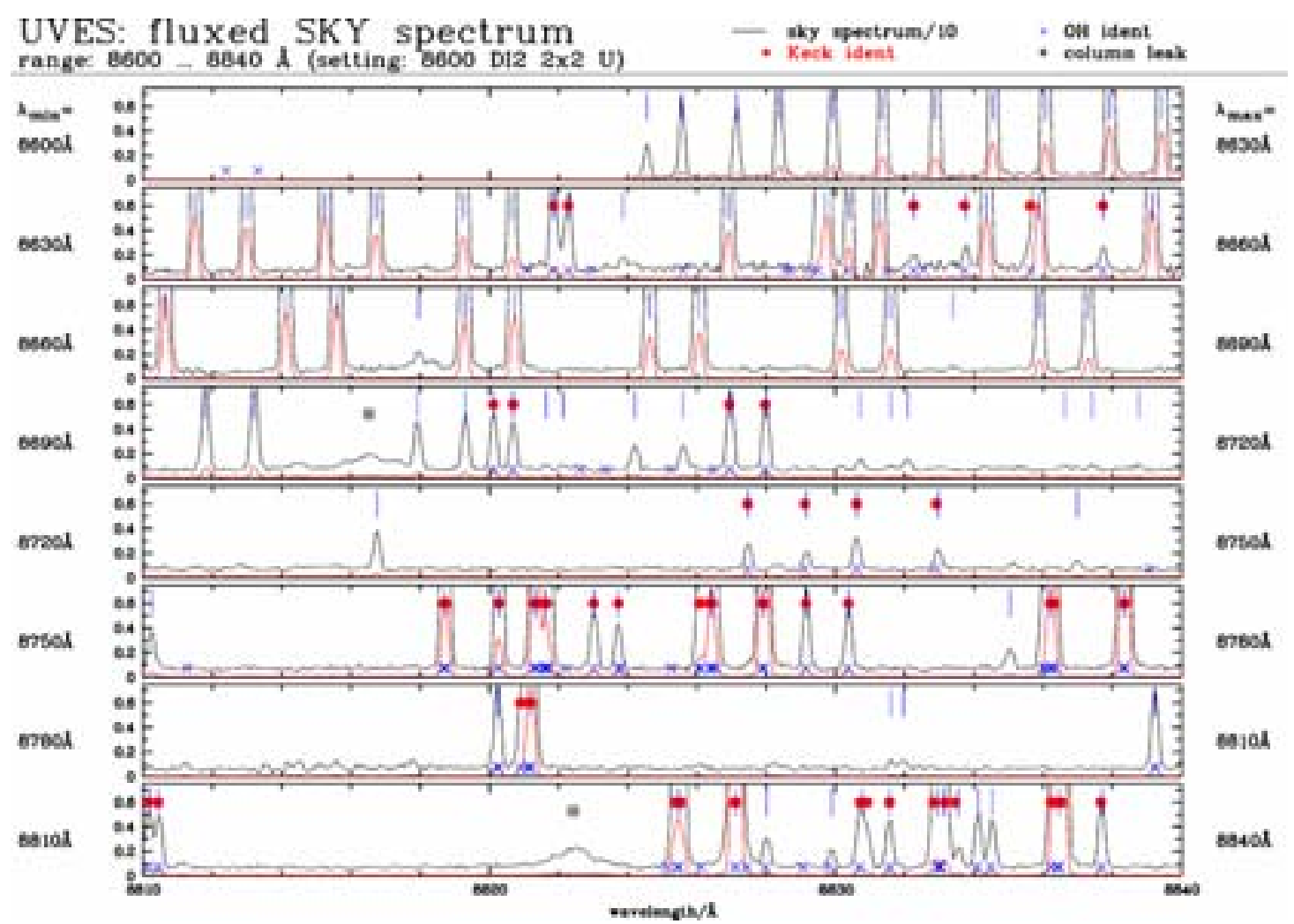

Fig. 34. UVES night-sky spectrum, covering 8600-8840 A. See the text of the Appendix for more general information about these plots. The vertical bars mark line measurements from this atlas. The sky spectrum is also plotted on a scale compressed by a factor 10 to better show stronger lines. The full circles mark emission lines with identification in the Keck atlas (Osterbrock et al. 1996). The crosses mark the (aircorrected) positions of identified OH lines in the Rousselot et al. (2000) and Lidman et al. (2000) lists. Artefacts due to a leaking column are marked by a squared plus. 
R. W. Hanuschik: Night-sky emission flux, Online Material p 16

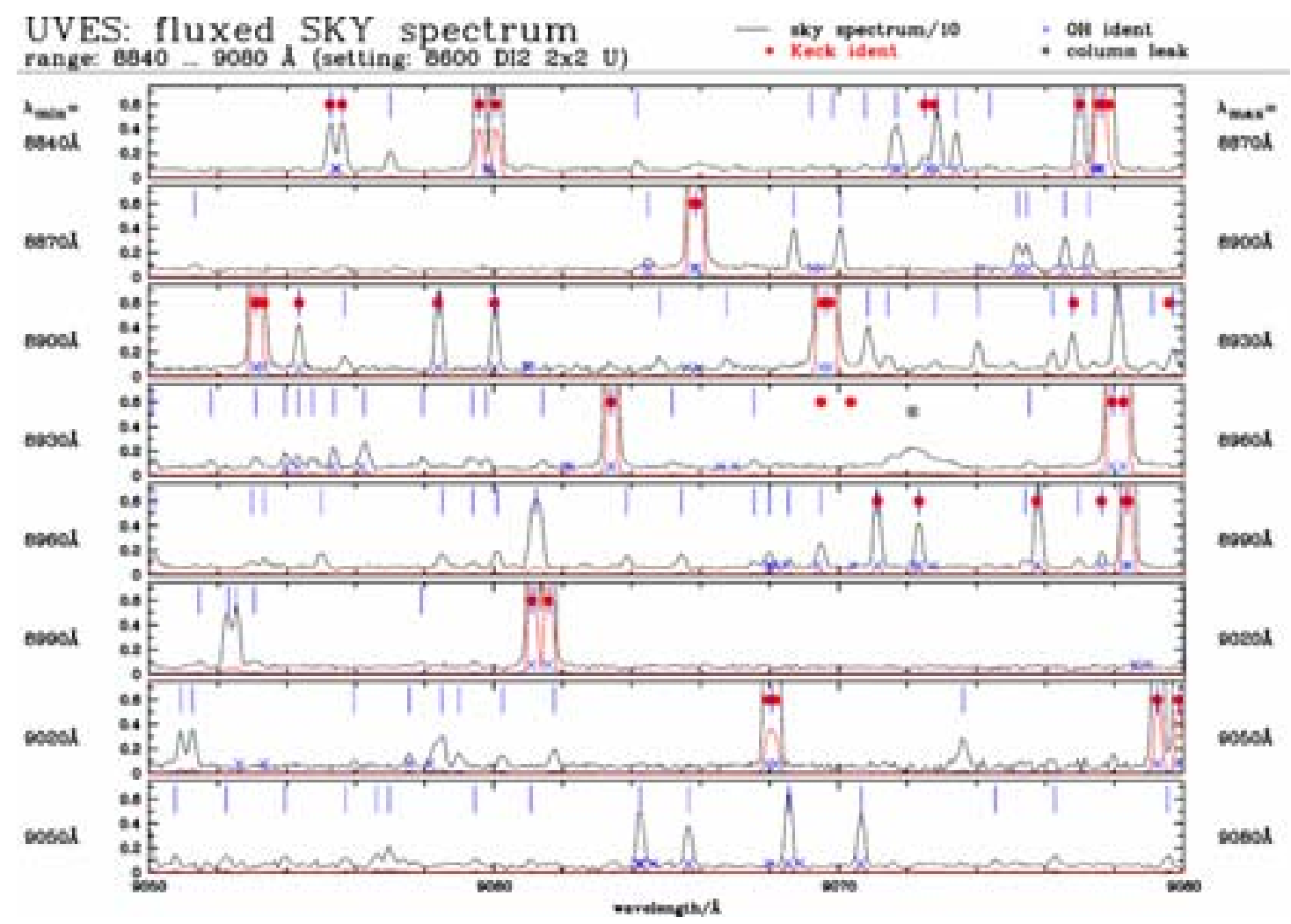

Fig. 35. Same as Fig. 34, for range 8840-9080 Å.

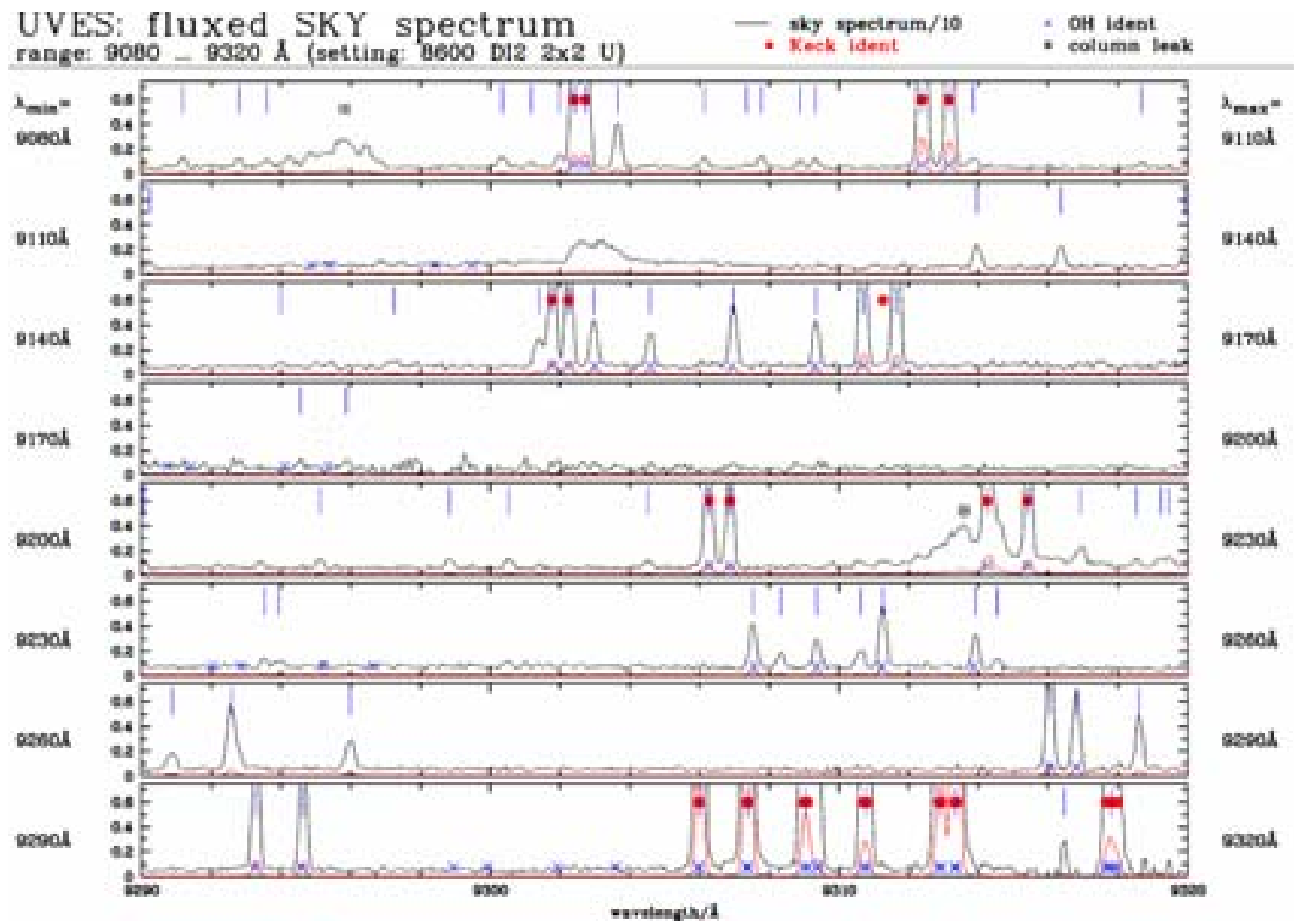

Fig. 36. Same as Fig. 34, for range 9080-9320 A. 
R. W. Hanuschik: Night-sky emission flux, Online Material p 17

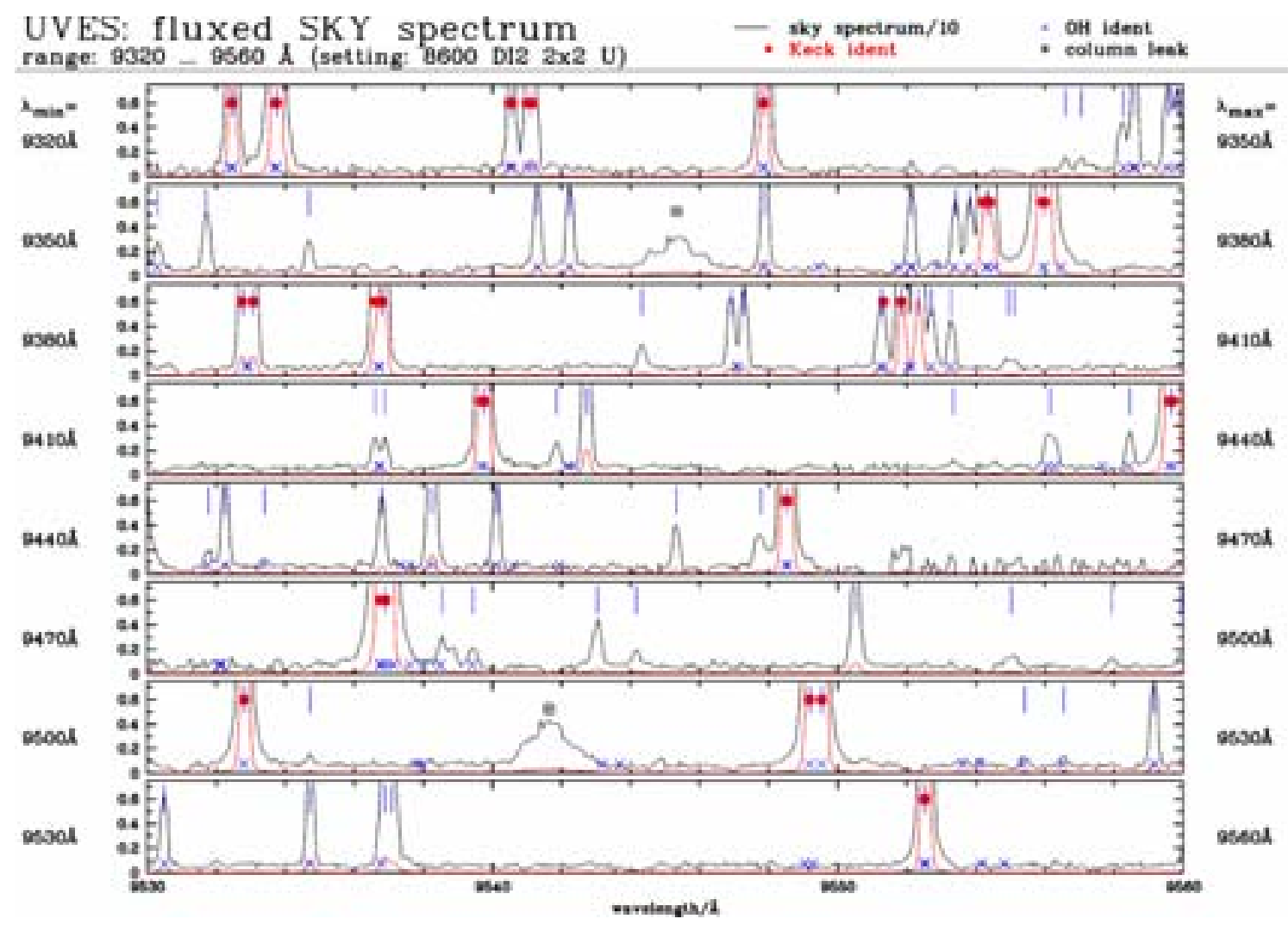

Fig. 37. Same as Fig. 34, for range 9320-9560 ̊̊.

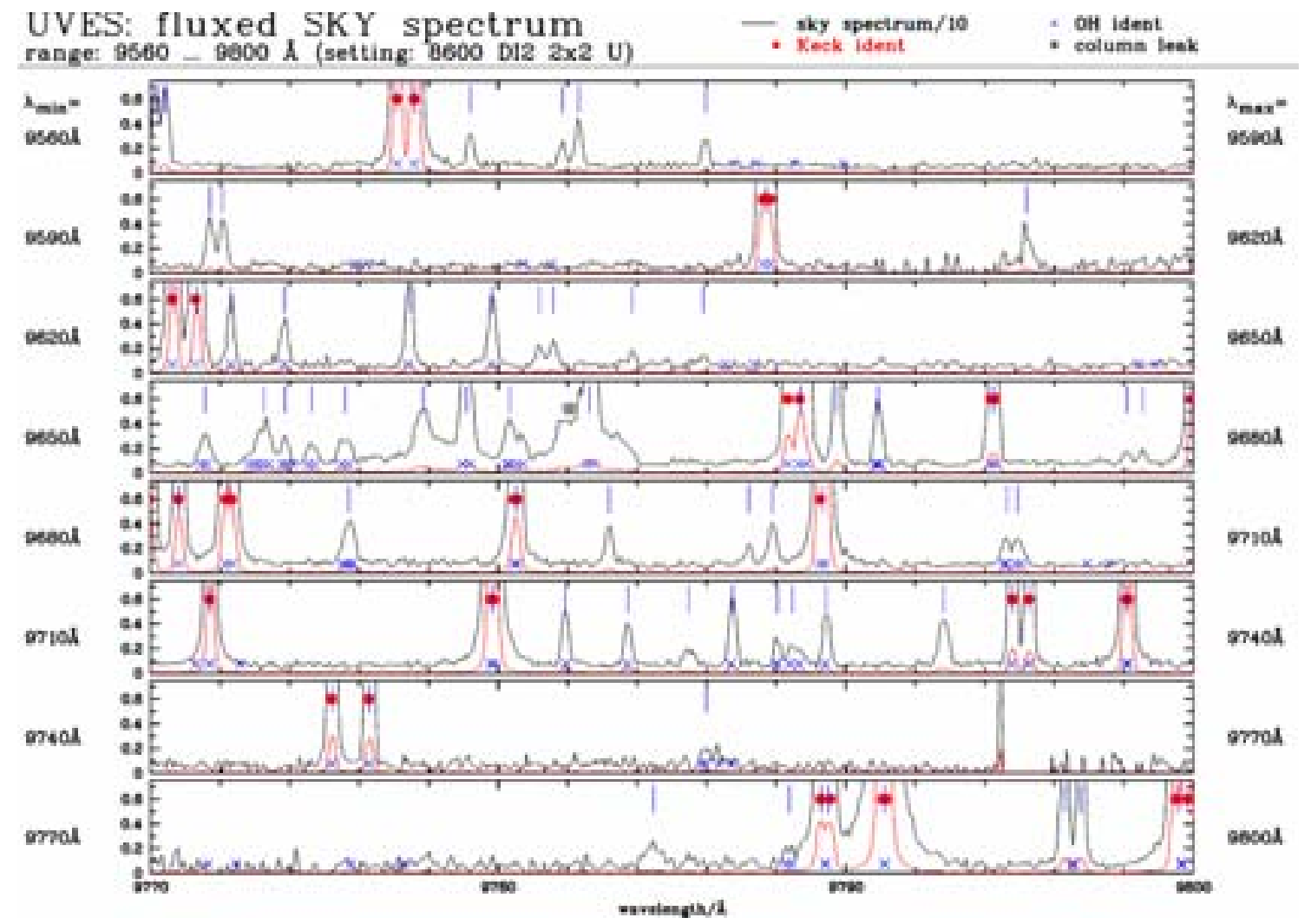

Fig. 38. Same as Fig. 34, for range 9560-9800 ̊. There is a small gap near $9765 \AA$, due to incomplete spectral coverage by the CCD. 
R. W. Hanuschik: Night-sky emission flux, Online Material p 18

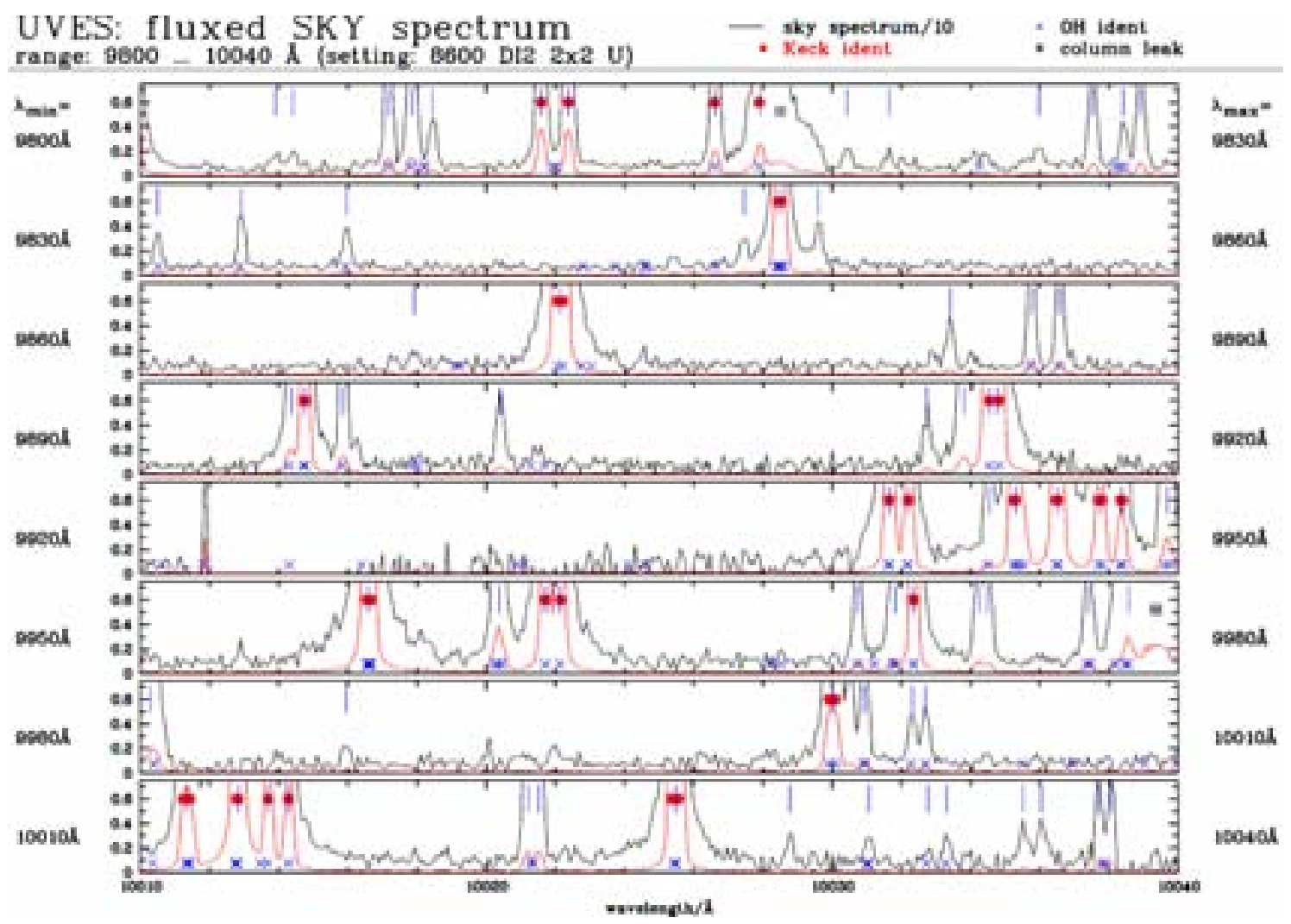

Fig. 39. Same as Fig. 34, for range 9800-10040 Å. There is a small gap near $9922 \AA$, due to incomplete spectral coverage by the CCD.

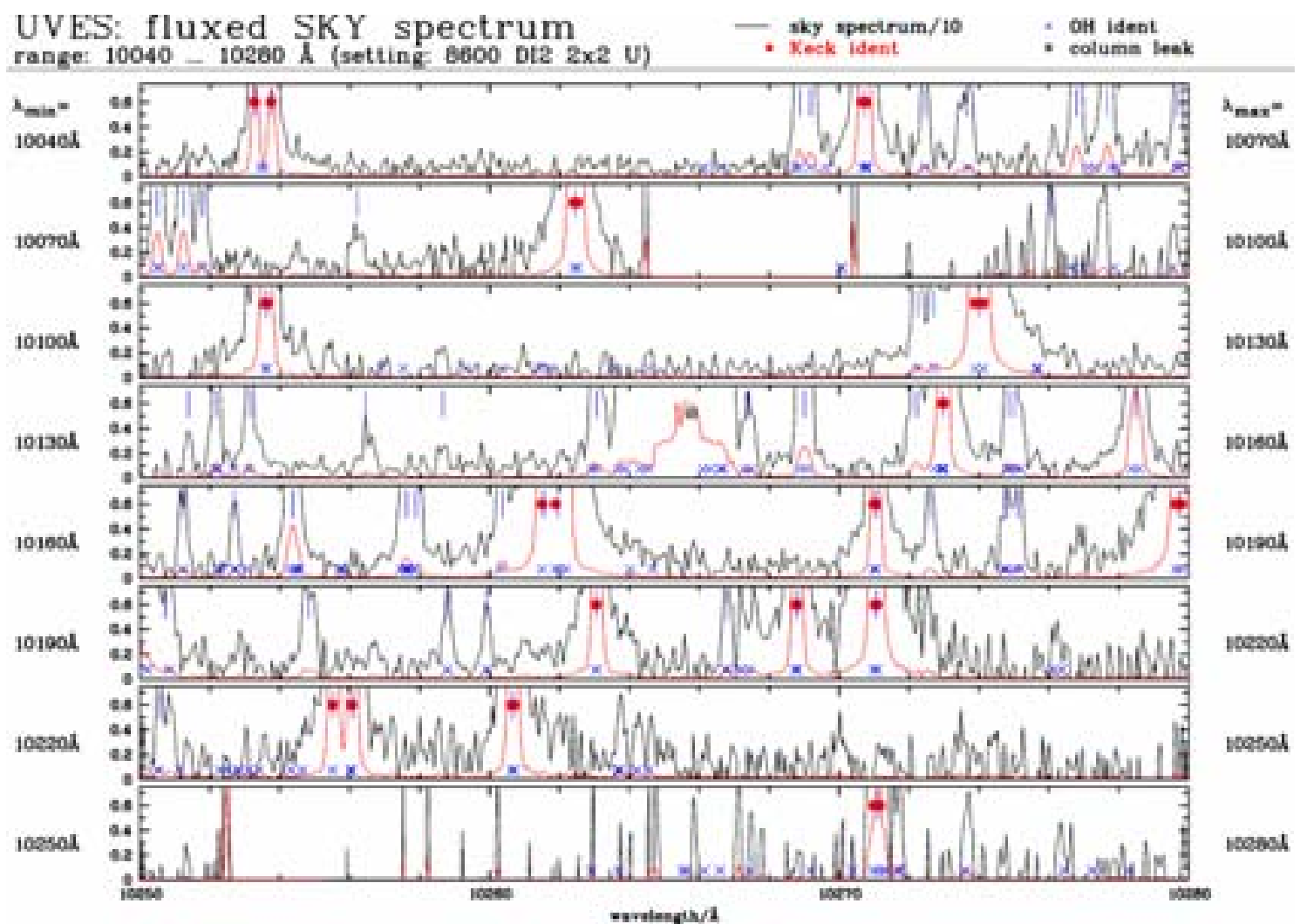

Fig. 40. Same as Fig. 34, for range 10040-10 $280 \AA$. There are two gaps near $10086 \AA$ and $10253 \AA$ due to incomplete spectral coverage by the CCD. 
R. W. Hanuschik: Night-sky emission flux, Online Material p 19

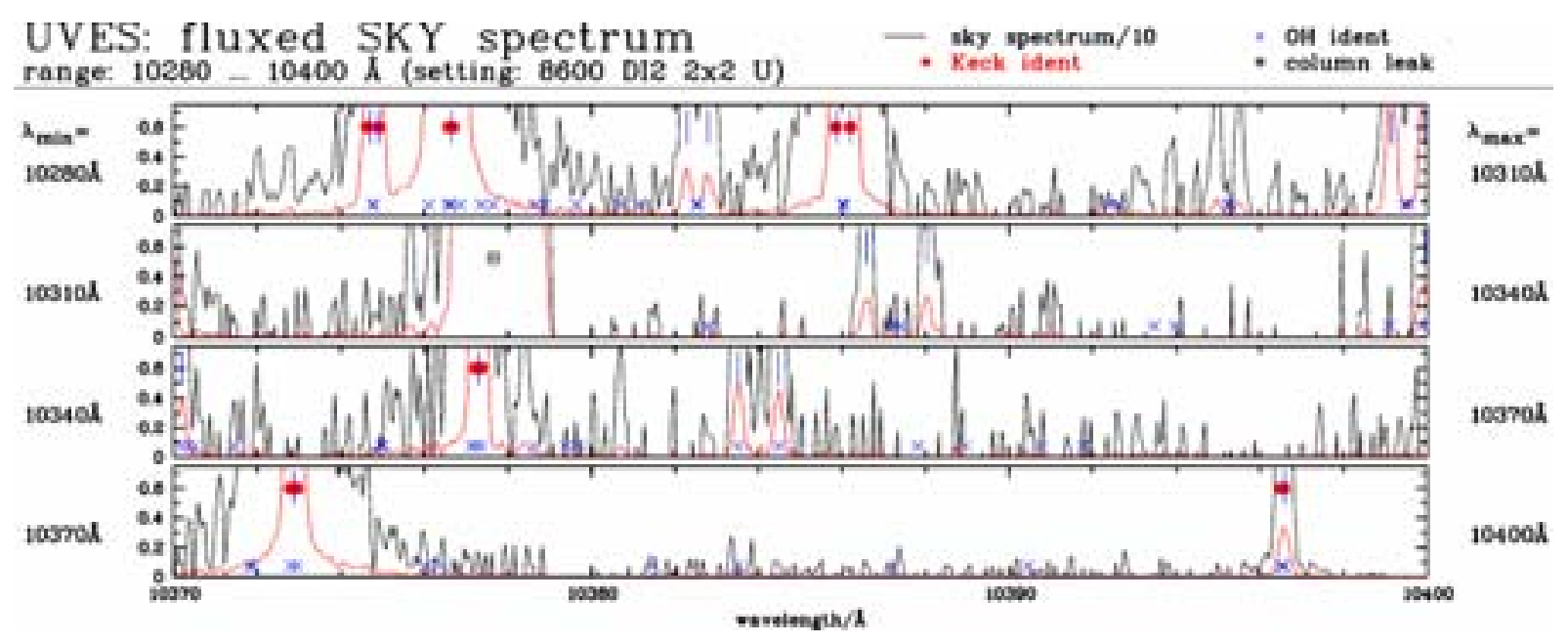

Fig. 41. Same as Fig. 34, for range 10 280-10 $430 \AA$ A. 